\title{
FTO-dependent demethylation of N6-methyladenosine regulates mRNA splicing and is required for adipogenesis
}

\author{
Xu Zhao ${ }^{1,2,{ }^{*}}$, Ying Yang ${ }^{1,2, *}$, Bao-Fa Sun ${ }^{1, *}$, Yue Shi ${ }^{1,2,{ }^{*}}$, Xin Yang ${ }^{1,2}$, Wen Xiao ${ }^{1,2}$, Ya-Juan Hao ${ }^{1,2}$, Xiao-Li Ping ${ }^{1,2}$, \\ Yu-Sheng Chen ${ }^{1,2}$, Wen-Jia Wang ${ }^{1,2}$, Kang-Xuan Jin ${ }^{1,2}$, Xing Wang ${ }^{1,2}$, Chun-Min Huang ${ }^{1}$, Yu Fu ${ }^{3}$, \\ Xiao-Meng Ge ${ }^{1}$, Shu-Hui Song ${ }^{1}$, Hyun Seok Jeong ${ }^{4}$, Hiroyuki Yanagisawa ${ }^{5}$, Yamei Niu ${ }^{6}$, Gui-Fang Jia ${ }^{7}, \mathrm{Wei}^{3}{ }^{3}$, \\ Wei-Min Tong ${ }^{6}$, Akimitsu Okamoto ${ }^{4,5}$, Chuan $\mathrm{He}^{7,8}$, Jannie M Rendtlew Danielsen ${ }^{1,9}$, Xiu-Jie Wang ${ }^{10}$, \\ Yun-Gui Yang ${ }^{1}$
}

${ }^{I}$ Key Laboratory of Genomic and Precision Medicine, Beijing Institute of Genomics, Chinese Acaemy of Sciences, No. 1-7 Beichen West Road, Chaoyang District, Beijing 100101, China; ${ }^{2}$ University of Chinese Academy of Sciences, 19A Yuquan Road, Beijing 100049, China; ${ }^{3}$ Protein Science Laboratory of the Ministry of Education, School of Life Sciences, Tsinghua University, Qinghuayuan 1, Beijing 100084, China; ${ }^{4}$ Research Center for Advanced Science and Technology, the University of Tokyo, 4-6-1 Komaba, Meguro-ku, Tokyo 153-8904, Japan; ${ }^{5}$ RIKEN Advanced Science Institute, 2-1 Hirosawa, Wako, Saitama 351-0198, Japan; ${ }^{6}$ Department of Pathology, Center for Experimental Animal Research, Institute of Basic Medical Sciences, Chinese Academy of Medical Sciences and Peking Union Medical College, Beijing 100005, China, ${ }^{7}$ Department of Chemical Biology, Beijing National Laboratory for Molecular Sciences, Synthetic and Functional Biomolecules Center, College of Chemistry and Molecular Engineering, Peking University, Beijing 100871, China; ${ }^{8}$ Department of Chemistry, Institute for Biophysical Dynamics, The University of Chicago, 929 East 57th Street, Chicago, IL 60637, USA; ${ }^{9}$ The Novo Nordisk Foundation Center for Protein Research, Ubiquitin Signalling Group, Faculty of Health Sciences, Blegdamsvej 3b, 2200 Copenhagen, Denmark; ${ }^{10}$ Key Laboratory of Genetic Network Biology, Institute of Genetics and Developmental Biology, Chinese Academy of Sciences, Beijing 100101, China.

The role of Fat Mass and Obesity-associated protein (FTO) and its substrate N6-methyladenosine $\left(\mathrm{m}^{6} \mathrm{~A}\right)$ in mRNA processing and adipogenesis remains largely unknown. We show that FTO expression and $\mathrm{m}^{6} \mathrm{~A}$ levels are inversely correlated during adipogenesis. FTO depletion blocks differentiation and only catalytically active FTO restores adipogenesis. Transcriptome analyses in combination with $\mathrm{m}^{6} \mathrm{~A}$-seq revealed that gene expression and $\mathrm{mRNA}$ splicing of grouped genes are regulated by FTO. $\mathrm{M}^{6} \mathrm{~A}$ is enriched in exonic regions flanking 5'- and $3^{\prime}$-splice sites, spatially overlapping with mRNA splicing regulatory serine/arginine-rich (SR) protein exonic splicing enhancer binding regions. Enhanced levels of $\mathbf{m}^{6} \mathrm{~A}$ in response to FTO depletion promotes the RNA binding ability of SRSF2 protein, leading to increased inclusion of target exons. FTO controls exonic splicing of adipogenic regulatory factor RUNX1T1 by regulating $\mathrm{m}^{6} \mathrm{~A}$ levels around splice sites and thereby modulates differentiation. These findings provide compelling evidence that FTO-dependent $\mathrm{m}^{6} \mathrm{~A}$ demethylation functions as a novel regulatory mechanism of RNA processing and plays a critical role in the regulation of adipogenesis.

Keywords: N6-methyladenosine ( $\left.\mathrm{m}^{6} \mathrm{~A}\right)$; METTL3; FTO; mRNA splicing; adipogenesis

Cell Research (2014) 24:1403-1419. doi:10.1038/cr.2014.151; published online 21 November 2014

*These four authors contributed equally to this work.

Correspondence: Yun-Gui Yang

Tel/Fax: +86-10-84097642

E-mail: ygyang@big.ac.cn

Received 30 June 2014; revised 23 September 2014; accepted 25 September 2014; published online 21 November 2014

\section{Introduction}

The Fat Mass and Obesity-associated (FTO) protein belongs to the AlkB family of non-heme Fe (II)/dioxygenases [1]. FTO is the first gene shown to contribute to non-syndromic human obesity [2-4]. In vivo studies using FTO overexpression or knockout mouse models have revealed abnormal adipose tissues and body mass, sug- 
gesting a pivotal role of FTO in adipogenesis and energy homeostasis [5-8]. In vitro FTO demethylates various methylated nucleic acids $[1,9,10]$. However, only demethylation of N6-methyladenosine $\left(\mathrm{m}^{6} \mathrm{~A}\right)$ in RNA has been confirmed in vivo [9]. First identified in mammalian mRNA in the $1970 \mathrm{~s}, \mathrm{~m}^{6} \mathrm{~A}$ has been proposed to regulate mRNA processing including alternative splicing, RNA degradation and translation [11-15]. In both mammals and yeast, $\mathrm{m}^{6} \mathrm{~A}$ modification of RNA preferentially occurs within the consensus sequence RRACH $(\mathrm{R}=\mathrm{G}$ or $\mathrm{A} ; \mathrm{H}=\mathrm{A}, \mathrm{C}$ or $\mathrm{U}$ ) in gene coding regions and $3^{\prime} \mathrm{UTR}$, implicating its fundamental roles in RNA processing and translational control [16-19]. There is a dramatic increase in $\mathrm{m}^{6} \mathrm{~A}$ levels during embryonic development and neuronal maturation in mammals and recent studies implicate that $\mathrm{m}^{6} \mathrm{~A}$ destabilizes developmental regulators in embryonic stem cells, and that the speed of the circadian clock is also regulated by $\mathrm{m}^{6} \mathrm{~A}$-dependent RNA processing [17, $18,20,21]$. In addition, in neuron-specific FTO-deficient mice, a subset of mRNAs involved in dopaminergic (DA) signaling shows increased $\mathrm{m}^{6} \mathrm{~A}$ levels [22]. FTO is most highly expressed in fetal tissues and in adult brain, adipose and muscle tissues. FTO deficiency results in a lean phenotype in mice $[5,23]$. However, the exact role of FTO in energy homeostasis and body weight control remains unclear [24]. Recently YTHDF2 was identified as an $\mathrm{m}^{6} \mathrm{~A}$-binding protein involved in the regulation of translation and stability of $\mathrm{m}^{6} \mathrm{~A}$-containing mRNAs [25]. The recent identification and characterization of the $\mathrm{m}^{6} \mathrm{~A}$ methyltransferase complex (METTL3-METTL14-WTAP) and its role in RNA splicing further highlight the biological significance of $\mathrm{m}^{6} \mathrm{~A}$ modification [26-28].

Splicing of mRNA precursors (pre-mRNA) to mature mRNAs is a highly dynamic and complex process influencing most biological functions in eukaryotes. RNA splicing is controlled by both cis-regulatory sequences within the RNA and trans-regulatory proteins. Two major classes of trans-acting factors, serine/arginine-rich (SR) proteins and heterogeneous ribonucleoproteins (hnRNPs), impact splice site recognition [29, 30]. Splice site selection is determined by cis-acting RNA sequences including intronic dinucleotides $\mathrm{GU}$ and $\mathrm{AG}$ (with variations used by the minor spliceosome) and the surrounding sequence context such as exonic/intronic splicing enhancers (E/ISEs) and silencers (E/ISSs) [31]. Other factors including factors modulating the chromatin and transcription complexes also affect RNA splicing [32]. Chemical inhibition of $\mathrm{m}^{6} \mathrm{~A}$ formation causes changes in the ratio between precursor and mature mRNAs [33], and $\mathrm{m}^{6} \mathrm{~A}$ has been observed to be significantly enriched in both multi-isoform genes and alternatively spliced exons [18]. Consistently, binding clusters of the $\mathrm{m}^{6} \mathrm{~A}$ methyltransferase components WTAP and METTL3 are preferentially present in genes with multiple isoforms [27]. Depletion of either METTL3 or ALKBH5 causes abnormal mRNA splicing as evident in genes in the p53 signaling pathways $[18,34]$. Speckle-localization of the $\mathrm{m}^{6} \mathrm{~A}$ methyltransferase WMM complex components as well as demethylases ALKBH5 and FTO further supports the role of $\mathrm{m}^{6} \mathrm{~A}$ in RNA processing [9, 27, 34]. ALKBH5 deficiency causes diminished SRSF1 (ASF/SF2) signal in the speckle [34]. Taken together, all these observations suggest a cis-regulatory element-dependent role of $\mathrm{m}^{6} \mathrm{~A}$ in RNA splicing.

The reported association of FTO activity with obesity prompted us to investigate how FTO activity influences RNA processing through regulation of $\mathrm{m}^{6} \mathrm{~A}$ abundance during adipogenesis. By combining transcriptome and $\mathrm{m}^{6} \mathrm{~A}$-seq analyses, we demonstrate that $\mathrm{m}^{6} \mathrm{~A}$ is enriched in exons adjacent to intronic $5^{\prime} / 3^{\prime}$ splice sites flanking spliced exons. In addition, we observe a striking spatial overlap of $\mathrm{m}^{6} \mathrm{~A}$ sites with mRNA exonic splicing enhancers recognized by SRSF2. Furthermore our results suggest that FTO controls mRNA splicing by regulating the RNA binding ability of SRSF2, and that FTO-regulated $\mathrm{m}^{6} \mathrm{~A}$ demethylation acts as a novel regulatory mechanism of RNA splicing, explaining its important role during adipogenesis.

\section{Results}

FTO-dependent $m^{6} \mathrm{~A}$ demethylation regulates adipogenesis

To investigate the function of $\mathrm{m}^{6} \mathrm{~A}$ modification in adipogenesis, we took advantage of the well-characterized mouse 3T3-L1 pre-adipocyte cell line (Supplementary information, Figure S1A). FTO, the other $\mathrm{m}^{6} \mathrm{~A}$ demethylase ALKBH5 or methyltransferase METTL3 was depleted individually by siRNA and then the pre-adipocytes were induced to differentiate (Figure 1A). Interestingly, FTO depletion severely impaired differentiation as demonstrated by Oil Red O staining (Figure 1B) and triglyceride content measurements (Figure 1C). This is consistent with decreased fat tissue accumulation observed in FTO-null mouse [5, 8, 35]. While METTL3 deficiency appeared to promote pre-adipocyte differentiation (Figure 1A-1C), ALKBH5 which is important for spermatogenesis [34], had no obvious effect on adipogenesis (Figure 1A-1C). Since FTO and METTL3 have opposing catalytic activities, their different effects on differentiation may be caused by changes in $\mathrm{m}^{6} \mathrm{~A}$ dynamics when either of them is depleted.

We next measured the expression of FTO and METTL3 during 3T3-L1 pre-adipocyte differentiation. Interestingly, the expression of FTO decreased during 
A

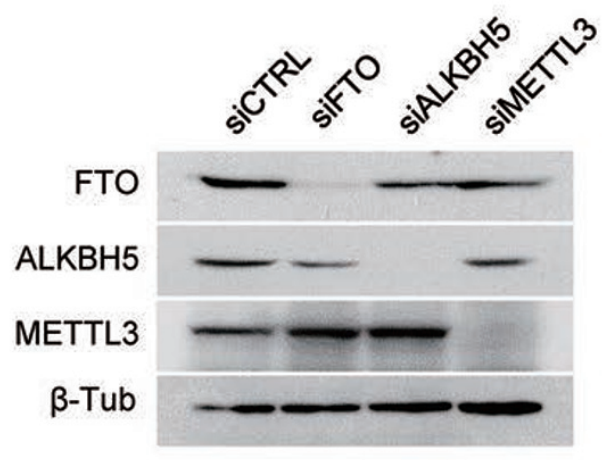

C

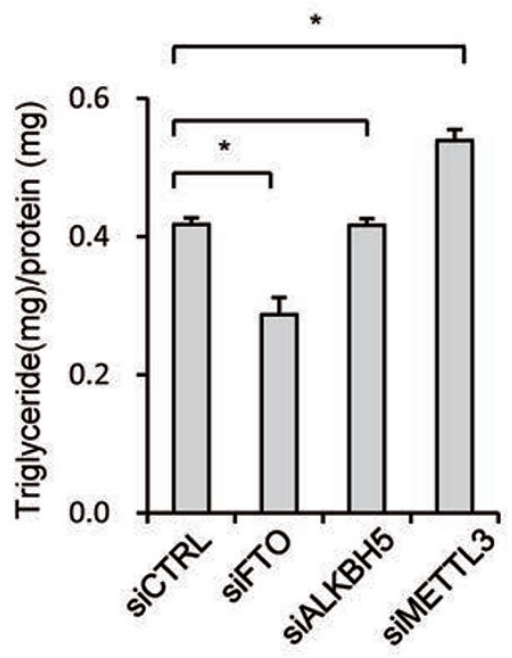

F

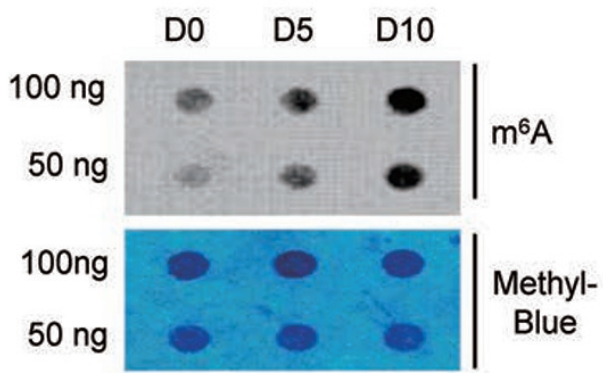

B

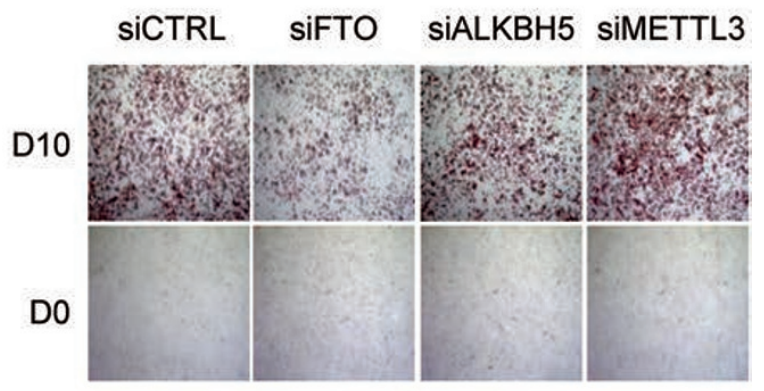

D

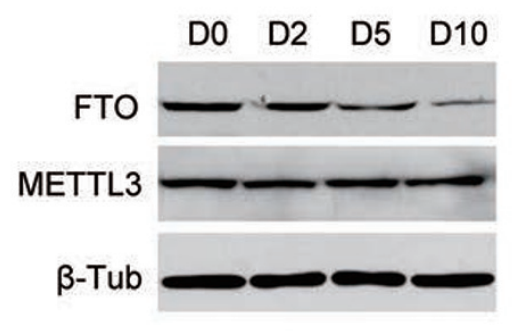

E

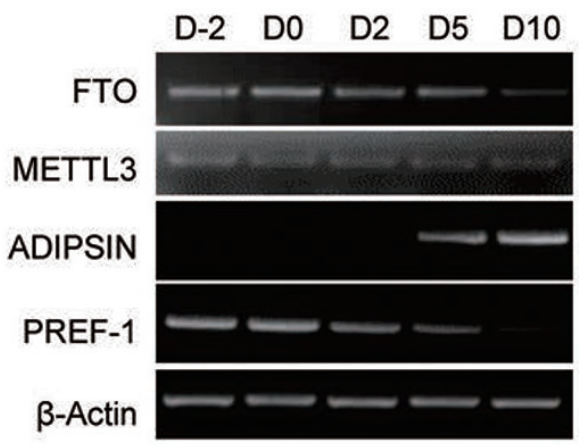

Figure 1 FTO depletion interferes with adipogenesis. (A-C) 3T3-L1 pre-adipocytes (Day -2) were treated with FTO, ALKBH5, METTL3 or control siRNA. Forty-eight hours later, cells were lysed and subjected to immunoblotting with the indicated antibodies (A). Forty-eight hours following siRNA treatment differentiation was induced by incubation with the differentiation cocktail (IBMX/DEX/Insulin) on Day 0. Differentiation status was determined by Oil Red O staining (B) and triglyceride assay (C) on Day 0 and 10. (C) Triglyceride content was quantified and normalized to protein content. ${ }^{*} P<0.05$ is considered significant. Results are shown as mean \pm SD. (D) 3T3-L1 cells collected at different time points (D0/2/5/10) during adipogenesis were lysed and subjected to immunoblotting with the indicated antibodies. $\beta$-tublin was used as loading control. (E) RT-PCR detected the expression levels of FTO, METTL3, as well as adipogenic markers, including ADIPSIN and PREF-1, during adipocyte differentiation. $\beta$-Actin was used as loading control. (F) mRNA was isolated from multiple stages (D0/5/10) of adipogenesis and used in dot blot analyses with $\mathrm{m}^{6} \mathrm{~A}$ antibody. mRNA was loaded by two-fold serial dilution. The $\mathrm{m}^{6} \mathrm{~A}$ contents are shown in the upper panel. Equal loading of mRNA was verified by methylene blue staining (lower panel). See also Supplementary information, Figure S1. 
differentiation and reached the lowest level in mature fat cells (D10) (Figure 1D, 1E and Supplementary information, Figure S1A), while the global $\mathrm{m}^{6} \mathrm{~A}$ levels were increased during the course of differentiation and reached the highest level in mature adipocytes (Figure 1F). METTL3 expression remained unchanged (Figure $1 \mathrm{D}$ and $1 \mathrm{E})$. Successful differentiation was evidenced by the characteristic increase in expression of the adipocyte marker, complement factor D (ADIPSIN), and decrease in expression of preadipocyte factor-1 (PREF-1) [36-38] (Figure 1E).

Differentiation of FTO-depleted pre-adipocytes could be fully restored by complementation with wild-type (WT) FTO but not by empty vector control or a FTO mutant protein in which three residues critical for FTO catalytic activity had been mutated [1] (H231A/D233A/ H307A, referred to as HDH; Supplementary information, Figure S1B-S1D). These results indicate that FTO regulates adipogenesis by modulating $\mathrm{m}^{6} \mathrm{~A}$ levels.

We next performed RNA-seq and $\mathrm{m}^{6} \mathrm{~A}$-seq analyses in control (siCTRL) and FTO-deficient (siFTO) 3T3L1 pre-adipocytes, as well as in 3T3-L1 pre-adipocytes at five stages (D -2/0/2/5/10) of adipogenesis. RNA-seq analysis was also performed in METTL3-depleted 3T3L1 cells. All of the cDNA libraries were sequenced using Hiseq 2000 (Illumina, $101 \mathrm{bp}$ ) and at least two biological replicates were performed for each experiment except for the experiments where we analyzed changes in five stages of adipogenesis. The deep sequencing data were mapped to mouse genome version $\mathrm{mm} 10$ without any gap and a maximum of two mismatches were allowed. The clean reads, mapped reads, uniquely mapped reads and mapping rates for all 22 samples are listed in Supplementary information, Table S1. Overall, 22-69 million uniquely mapped reads with mapping quality higher than or equal to 20 were obtained for these samples and their mapping rates ranged from $72 \%$ to $90 \%$. The $\mathrm{m}^{6} \mathrm{~A}$ regions $\left(\mathrm{m}^{6} \mathrm{~A}\right.$ peaks) were identified by comparing the read abundance between $\mathrm{m}^{6} \mathrm{~A}$-Seq and RNA-seq samples of the same locus according to a method previously described [17]. In total, 8710 and $10137 \mathrm{~m}^{6} \mathrm{~A}$-modified genes in control (siCTRL) and FTO-depleted (siFTO) cells with significant $\mathrm{m}^{6} \mathrm{~A}$ enrichment were identified, respectively (Supplementary information, Table S2). This is consistent with the overall increase of $\mathrm{m}^{6} \mathrm{~A}$ levels in FTO-depleted cells compared with control cells as observed by dot blot analysis (Supplementary information, Figure S2A). The $\mathrm{m}^{6} \mathrm{~A}$ distribution profiles and motifs are similar to previous observations [17, 18], verifying the successful enrichment of $m^{6} \mathrm{~A}-\mathrm{mRNAs}$ by $\mathrm{m}^{6} \mathrm{~A}$-IP (Supplementary information, Figure S2B and S2C). Recent reports demonstrate that the METTL3-METTL14-WTAP complex is responsible for N6-methylation of adenosine in mRNA [26-28]. Therefore, we speculated that FTO and METTL3 might dynamically regulate mRNA $\mathrm{m}^{6} \mathrm{~A}$ levels and gene expression. We found that the expression of 5071 genes was affected by FTO depletion, while 6 065 genes were altered by METTL3 depletion. By overlapping differentially expressed genes (DEGs) upon FTO and METTL3 knockdown, we found that 524/553 genes were up/downregulated by FTO depletion while down/ upregulated by METTL3 depletion (Supplementary information, Figure S2D). 428 of these $1077(524+553)$ genes showed increased $\mathrm{m}^{6} \mathrm{~A}$ levels upon FTO depletion, suggesting that $\mathrm{m}^{6} \mathrm{~A}$ modification in this subset of genes is co-regulated by FTO and METTL3 (Supplementary information, Figure S2E and S2F). To investigate the $\mathrm{m}^{6} \mathrm{~A}$ distribution patterns within these 428 genes in more detail we divided each transcript into 5'-UTR, CDS and 3'-UTR regions. 174 genes showed no changes in overall $\mathrm{m}^{6} \mathrm{~A}$ distribution pattern but higher $\mathrm{m}^{6} \mathrm{~A}$ levels within individual peaks while in the other 254 genes, 337 new $\mathrm{m}^{6} \mathrm{~A}$ peaks were identified. New $\mathrm{m}^{6} \mathrm{~A}$ sites were mainly located in CDS (179) and 3'-UTR (135) regions and $\sim 90 \%$ of the modifications were located in RRACH consensus motifs (Supplementary information, Figure S2G). As expected, only $74 \mathrm{~m}^{6} \mathrm{~A}$ peaks disappeared upon FTO depletion and 63 of them were located in $5^{\prime}$-UTR regions.

Gene ontology (GO) analysis revealed that the 428 co-regulated genes were enriched in lipid synthesis, protein localization and transport, cellular movement and translation-related pathways, suggesting that FTO and METTL3 might co-regulate a group of functionally diverse genes (Supplementary information, Figure $\mathrm{S} 2 \mathrm{H}$ ).

Next we characterized the $\mathrm{m}^{6} \mathrm{~A}$ dynamics during adipogenesis by performing $\mathrm{m}^{6} \mathrm{~A}$-seq at five stages (D-2/0/2/5/10) of adipogenesis. 11427 genes with $\mathrm{m}^{6} \mathrm{~A}$ were observed at the five stages and 6313 of them were found to contain $\mathrm{m}^{6} \mathrm{~A}$ modification throughout the five stages (Supplementary information, Figure S3A). The GGAC (A/U) motif with $\mathrm{m}^{6} \mathrm{~A}$ modification was highly enriched at all stages (Supplementary information, Figure S3B). Overall, $\mathrm{m}^{6} \mathrm{~A}$ level was inversely associated with gene expression as illustrated in the heatmap (Supplementary information, Figure S3C). Genes highly expressed at early stages of differentiation were associated with regulation of transcription, cellular metabolic processes and cell growth, while highly expressed genes in fat cells were involved in the regulation of adipocyte differentiation and oxidation reduction processes (Supplementary information, Figure S3C).

FTO-dependent demethylation of $m^{6} \mathrm{~A}$ controls $R N A$ 
splicing

Previous findings have shown that $\mathrm{m}^{6} \mathrm{~A}$ demethylases and members of the $\mathrm{m}^{6} \mathrm{~A}$ methyltransferase complex are localized in nuclear speckles, suggesting a potential role of $\mathrm{m}^{6} \mathrm{~A}$ in RNA splicing $[9,27,34,39]$. Analyses by Cufflink showed that 279 out of 694 genes without obvious changes in gene expression levels (fold change $<2$ ) but with significant changes in the main isoforms (fold change $>2$ ), were $\mathrm{m}^{6} \mathrm{~A}$-modified $(P=2.2 \mathrm{e}-10$, hypergeometric test). Scripture analysis showed that 6 329 of 7374 genes with differently spliced isoforms were $\mathrm{m}^{6} \mathrm{~A}$-modified $(P<1 \mathrm{e}-305$, hypergeometric test). When comparing single- and multi-isoform genes with the Ensembl-annotated reference (background), it was evident that $\mathrm{m}^{6} \mathrm{~A}$ is more common in multi-isoform genes compared to single-isoform genes (Figure 2A). In addition, the average number of $\mathrm{m}^{6} \mathrm{~A}$ peaks per gene was also higher in multi-isoform genes (4.52 peaks per gene) compared to single-isoform genes (3.65 peaks per gene). At the exon/intron level, 376 of 798 differentially expressed exons $(P=3.9 \mathrm{e}-27$, hypergeometric test $)$ and 1613 of 3521 differentially expressed introns $(P=$ 2.6e-105, hypergeometric test) bare $\mathrm{m}^{6} \mathrm{~A}$. Comparing the $\mathrm{m}^{6} \mathrm{~A}$-modified exons with Ensembl-annotated genomic exons, we found that $51 \%$ of the constitutive exons (CNE) were $\mathrm{m}^{6} \mathrm{~A}$-modified, which is $14 \%$ less than the $65 \%$ predicted by the Ensembl-annotation $(P<2.2 \mathrm{e}-16$, Fisher's exact test). In contrast, $\mathrm{m}^{6} \mathrm{~A}$ was overrepresented in both alternative cassette (CE) exons and intron retention (IR) splicing events, and $\mathrm{m}^{6} \mathrm{~A}$ peaks within $\mathrm{CE}$ exons increased upon FTO depletion $(P<0.01, t$-test) (Figure 2B), suggesting that FTO directly regulates $\mathrm{m}^{6} \mathrm{~A}$ levels during this type of splicing.

We next used Cufflink to calculate the $\mathrm{m}^{6} \mathrm{~A}$ level of each isoform using the FPKM (fragments per kilobase of transcript per million mapped reads) method to make the estimate. When comparing the isoform changes upon FTO and METTL3 depletion, an inverse expression pattern was observed in $1491(611+880)$ isoforms of 1335 genes $(580+755)$ (Figure $2 \mathrm{C})$. Of these, 452 genes $(522$ isoforms) showed increased $\mathrm{m}^{6} \mathrm{~A}$ levels (in either peak number or peak enrichment) following FTO knockdown (Figure $2 \mathrm{D}$ and $2 \mathrm{E}$ ), suggesting that $\mathrm{m}^{6} \mathrm{~A}$ may directly influence isoform expression in this subset of genes. Analysis of $\mathrm{m}^{6} \mathrm{~A}$ distribution in $5^{\prime}$-UTR, CDS and $3^{\prime}$-UTR segments revealed that 214 of the 452 co-regulated genes contained conserved $\mathrm{m}^{6} \mathrm{~A}$ sites, while 332 new $\mathrm{m}^{6} \mathrm{~A}$ peaks had occurred in the other 238 genes upon FTO depletion (Figure 2F). GO analysis showed enrichment of genes with functions related to sterol metabolic processes and organelle organization (Figure 2G). During the course of adipogenesis, we identified 17637 significantly differentially expressed isoforms. $\mathrm{M}^{6} \mathrm{~A}$ levels at different stages were illustrated in a heatmap (Supplementary information, Figure S3D). A representative gene FBXO9 displays increased $\mathrm{m}^{6} \mathrm{~A}$ levels in FTO-depleted 3T3-L1 cells and progressively rising $\mathrm{m}^{6} \mathrm{~A}$ levels during adipocyte differentiation (Supplementary information, Figure S4).

\section{$M^{6} A$ sites spatially overlap with $5^{\prime}$ and $3^{\prime}$ exonic flanking sequences}

Cis-elements found within exons, such as exonic splicing enhancers (ESEs) and exonic splicing silencers (ESSs) in the vicinity of splice sites (both $5^{\prime}$ and $3^{\prime}$ splice sites), play important roles in regulating inclusion or skipping of exons [40]. In addition, in human and mouse the conserved intronic sequences flanking both sides of an alternatively spliced exon have also been proposed to be involved in the regulation of alternative splicing [41, 42]. The findings presented so far in combination with previous reports suggest the possible involvement of $\mathrm{m}^{6} \mathrm{~A}$ in the regulation of RNA splicing $[18,27]$. To further test this, we examined the distribution of $\mathrm{m}^{6} \mathrm{~A}$ sites in the vicinity of splice sites. Using procedures reported previously [43], we analyzed $\mathrm{m}^{6} \mathrm{~A}$ peak distributions in mRNAs within 100-300 nt upstream or downstream of the constitutive splice sites. Meanwhile, to analyze the effect of FTO depletion on $\mathrm{m}^{6} \mathrm{~A}$ peak number and enrichment, total $\mathrm{m}^{6} \mathrm{~A}$ enrichments for $\mathrm{m}^{6} \mathrm{~A}$ peaks located within $100 \mathrm{nt}$ up- and downstream of $5^{\prime}$ and $3^{\prime}$ splice sites were calculated, respectively. For 5326 genes showing changes in isoform expression upon FTO depletion, a significant overrepresentation of $\mathrm{m}^{6} \mathrm{~A}$ sites in $5^{\prime}$ and $3^{\prime}$ exonic sequences flanking the constitutive splice site was observed when compared to intronic sequences adjacent to the splice site (Figure 3A and Supplementary information, Table S3). FTO deficiency further increased the $\mathrm{m}^{6} \mathrm{~A}$ enrichment in these regions (Figure $3 \mathrm{~A}$, lower panel and Supplementary information, Table S3). For the 522 isoforms (452 genes) that were inversely affected in expression levels by FTO and METTL3 depletion and showed increased $\mathrm{m}^{6} \mathrm{~A}$ levels upon FTO depletion, the effect of FTO depletion on $\mathrm{m}^{6} \mathrm{~A}$ levels in regions surrounding constitutive splice sites was even more pronounced (5' exon, 2.50-fold; $3^{\prime}$ exon, 2.68-fold; Figure 3B, lower panel). Similar results regarding $\mathrm{m}^{6} \mathrm{~A}$ distribution pattern and enrichment were obtained around alternative splice sites (Figure 3C and 3D). On the whole, although FTO depletion barely affected the overall distribution pattern of $\mathrm{m}^{6} \mathrm{~A}$ peaks, it increased the $\mathrm{m}^{6} \mathrm{~A}$ levels at individual $\mathrm{m}^{6} \mathrm{~A}$ peaks (Figure 3A-3D). We next analyzed previously published $\mathrm{m}^{6} \mathrm{~A}-\mathrm{Seq} / \mathrm{MeRIP}-\mathrm{Seq}$ datasets $[17,18]$. Consistently, in these datasets the majority of $\mathrm{m}^{6} \mathrm{~A}$ peaks in 
A

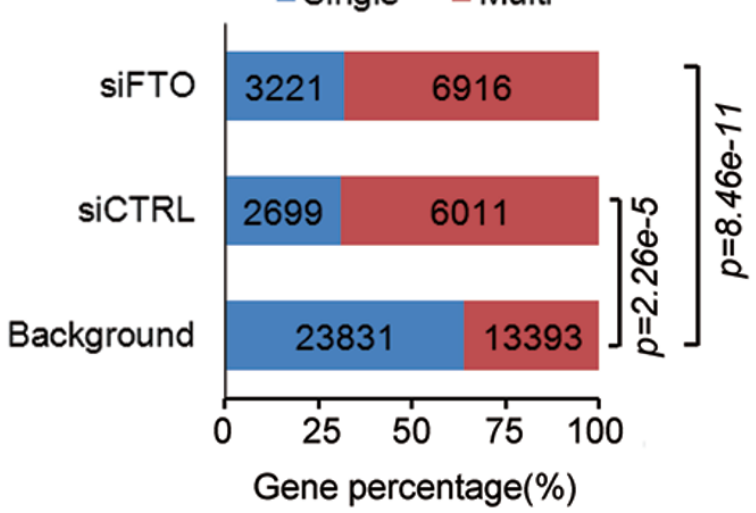

C

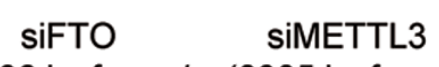

(9536 isoforms/ (8905 isoforms/

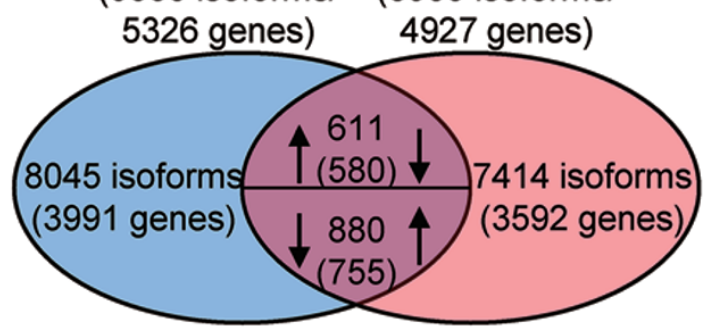

E

Log2 (Relative fold change to SiCTRL)

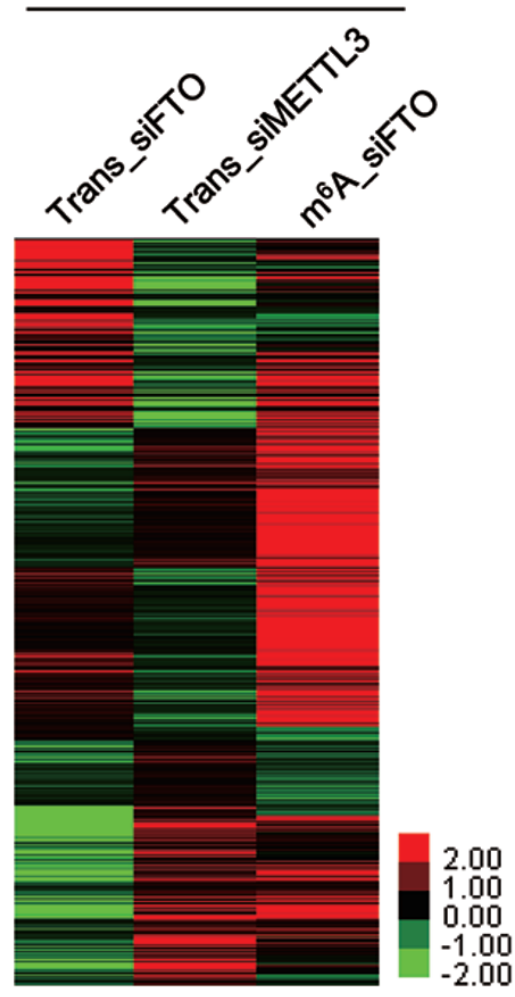

B

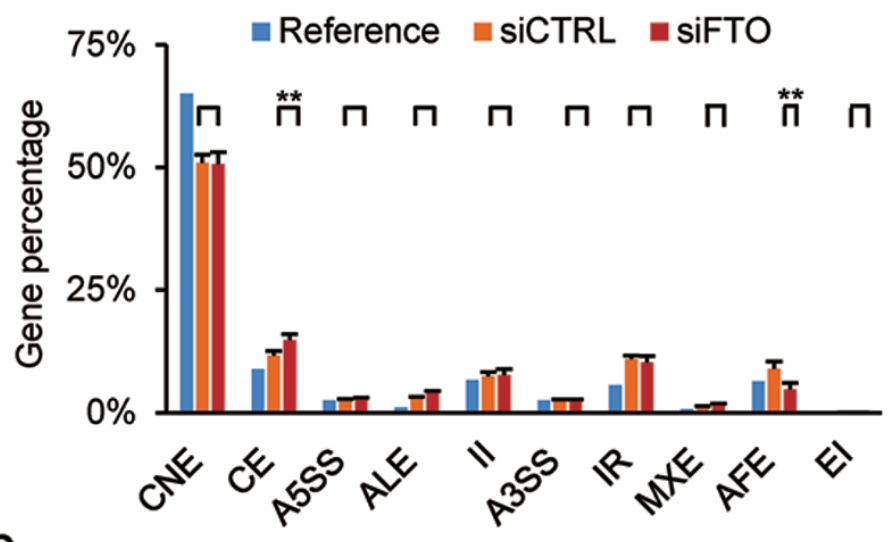

D

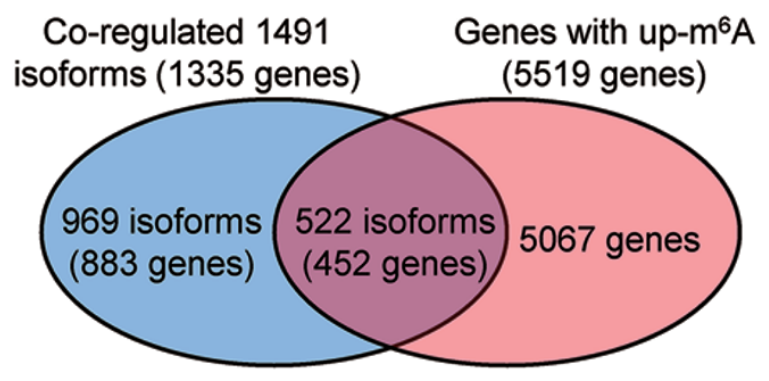

$\mathbf{F}$

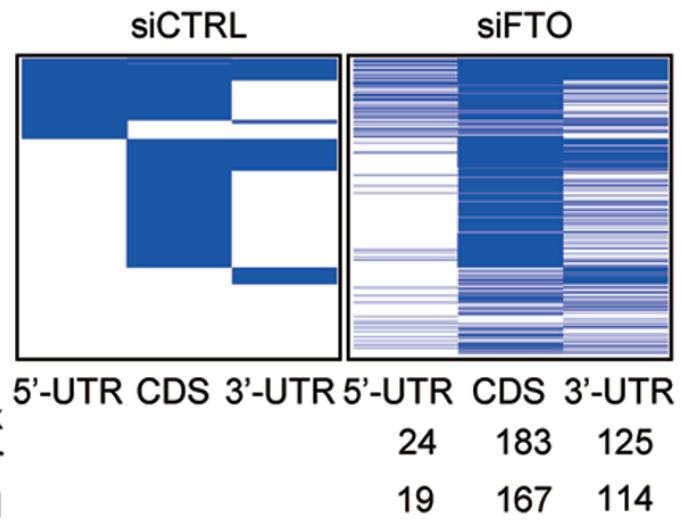

G

New peak

Number

$\mathrm{RRACH}$

$19 \quad 167 \quad 114$

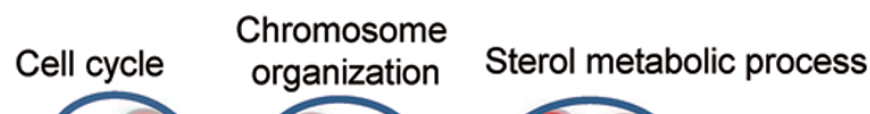

Regulation of organelle organization

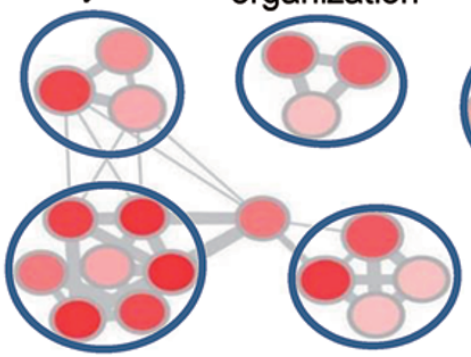

Protein localization

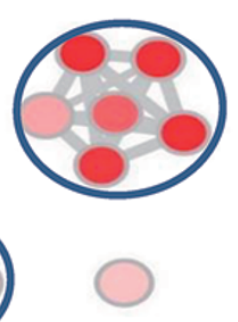

Vesicle-mediated transport 
exons were also mainly located in regions adjacent to constitutive and alternative splice sites (Supplementary information, Figure S5A).

The spatial overlap of $\mathrm{m}^{6} \mathrm{~A}$ with constitutive/alternative splice sites was observed at all stages of adipogenesis (Supplementary information, Figure S5B-S5E). Consistent with our above findings that the global $\mathrm{m}^{6} \mathrm{~A}$ levels increased while FTO expression levels decreased during the course of adipocyte differentiation (Figure 1D and $1 \mathrm{E})$, a minor increase in $\mathrm{m}^{6} \mathrm{~A}$ levels around splice sites was evident in more differentiated cells (Supplementary information, Figure S5B-S5E). Taken together, these observations strongly support a regulatory role of $\mathrm{m}^{6} \mathrm{~A}$ in RNA splicing.

$M^{6}$ A sites show a spatial overlap with SRSF1- and 2-binding clusters

SR proteins are important for splice site recognition and intron processing. They recognize the pre-mRNA cis-acting elements, ESEs, and thereby participate in the selection of splice sites. ESEs recognized by SRSF1 and 2 , like $\mathrm{m}^{6} \mathrm{~A}$, are highly enriched at the edges of exons [44], suggesting a potential cooperative role between SRSF1/2 and $\mathrm{m}^{6} \mathrm{~A}$ in RNA splicing. To test this, we calculated the distances between SRSF1-4-binding clusters based on the previously published CLIP-seq datasets $[45,46]$ and the nearest $\mathrm{m}^{6} \mathrm{~A}$ sites. As a control, randomly selected sites with the same length as the $\mathrm{m}^{6} \mathrm{~A}$ peak within the same exons were generated by BEDTools' shuffleBed. There was a significant spatial overlap between SRSF1and 2-binding clusters and $\mathrm{m}^{6} \mathrm{~A}$ sites (Figure 4A). In contrast, no overlap between SRSF3, 4 or ribonucleoproteins hnRNPC/hnRNRH $[47,48]$ and $\mathrm{m}^{6} \mathrm{~A}$ sites was detected (Figure 4A and Supplementary information, Figure S6A-
S6D). PhyloP scores across 60 vertebrates [49] generated by comparing the specific sites with random exonic regions of the same size indicated a significant conservation of the overlapping $\mathrm{m}^{6} \mathrm{~A}$ and SRSF1- and 2-binding sites $(P=2.2 \mathrm{e}-16$, Kolmogorov-Smirnov test; Supplementary information, Figure S7A). Furthermore, the potential SRSF1- or 2-recognized ESEs identified from m6A-modified exons by ESEfinder $[50,51]$ significantly overlapped with m6A sites. These predicted ESEs were also enriched with the RRACH motif found in $\mathrm{m}^{6} \mathrm{~A}$ sites (Figure 4B). In details, $60 \%-80 \%$ of the predicted ESEs overlapped with m6A sites $(P=2.2 \mathrm{e}-16$, Fisher test $)$ and $20 \%-30 \%$ overlapped with the RRACH motif $(P=2.2 \mathrm{e}$ 16 , Fisher test). Collectively, these results suggest that $\mathrm{m}^{6} \mathrm{~A}$ might specifically influence the binding of SRSF 1 and 2 to ESEs.

Consistently, like $\mathrm{m}^{6} \mathrm{~A}$, we found that SRSF1- and 2-binding clusters were strongly enriched around splice sites, while SRSF3 and 4 binding patterns were much less pronounced (Figure 4C). The mRNA binding of both hnRNPC and hnRNRH was decreased at the splice sites (Supplementary information, Figure S6A-S6D). Interestingly, in addition to the known GGAGA motif for SRSF1 and GA-rich motif for SRSF2 [45] binding, we found that $\mathrm{m}^{6} \mathrm{~A}$ motifs can also serve as SRSF1- (UGGAC, $P$ $=1 \mathrm{e}-547$; AGGACCU, $P=1 \mathrm{e}-99$ ) and 2- (UGGAC, $P=$ 1e-1207; AAGGACC, $P=1 \mathrm{e}-182$ ) binding sites, but not SRSF3- or 4-binding sites (Supplementary information, Figure S7B).

Using SRSF2 as an example, we next tested the effects of FTO depletion on its RNA binding ability. Interestingly, FTO knockdown promoted the RNA binding ability of SRSF2 (Figure 4D and 4E), but had no obvious effect on that of SRSF4 (Supplementary information,

Figure 2 FTO affects RNA splicing via modulating $\mathrm{m}^{6} \mathrm{~A}$. (A) The percentage of $\mathrm{m}^{6} \mathrm{~A}-\mathrm{mRNAs}$ in control $(6011$ multi-isoform/2 699 single-isoform, $P=2.26 \mathrm{e}-5$, Fisher test) and FTO-knockdown cells (6 916 multi-isoform/3 221 single-isoform, $P=8.46 \mathrm{e}-$ 11 , Fisher test) derived from single-isoform or multi-isoform genes compared to the distribution predicted by the Ensembl-annotation reference (background). (B) The distribution of $\mathrm{m}^{6} \mathrm{~A}$-containing exons across different categories of splicing events in both control (orange) and FTO-depleted samples (red) compared to that Ensembl-annotation reference (Blue). $P$ value for each category was calculated between siCTRL and siFTO samples. ${ }^{* *} P<0.01$ (Student's $t$-test) is considered significant $(n=2)$. Results are shown as mean \pm SD. CNE, constitutive exon; CE, cassette exon; A5SS, alternative 5' splice site; ALE, alternative last exon; II, intron isoform; A3SS, alternative 3' splice site; IR, intron retention; MXE, mutually exclusive exons; AFE, alternative first exon; El, exon isoform. (C) 1491 isoforms (1 335 genes) are co-regulated by FTO and METTL3. Arrows pointing up/down indicates up/down-regulation. (D) FTO depletion resulted in increased $\mathrm{m}^{6} \mathrm{~A}$ levels in 522 isoforms (452 genes) of the 1491 isoforms (1 335 genes) co-regulated by FTO and METTL3 (shown in C). (E) The heatmap shows the expression levels of 522 reverse-regulated isoforms by FTO and METTL3, as well as the $\mathrm{m}^{6} \mathrm{~A}$ modification in FTO-depleted cells. (F) The heatmap shows $\mathrm{m}^{6} \mathrm{~A}$ peaks in 5'-UTR, CDS, and 3'-UTR (522 isoforms co-regulated by FTO and METTL3) in control and FTO-deficient cells. Blue lines represent $\mathrm{m}^{6} \mathrm{~A}$ peaks. Each horizontal line represents one gene. The number of new $\mathrm{m}^{6} \mathrm{~A}$ peaks upon FTO depletion and new $\mathrm{m}^{6} \mathrm{~A}$ peaks within a RRACH motif are shown below the heatmap. (G) Function enrichment analysis of 522 isoforms (452 genes) based on the DAVID GO analysis result. The cutoff parameters for enrichment analysis with Cytoscape software are: $P<0.005$, FDR $q<0.1$, overlap cutoff $>0.5$. See also Supplementary information, Figures S2-S4. 
A

Total genes (5326 genes)

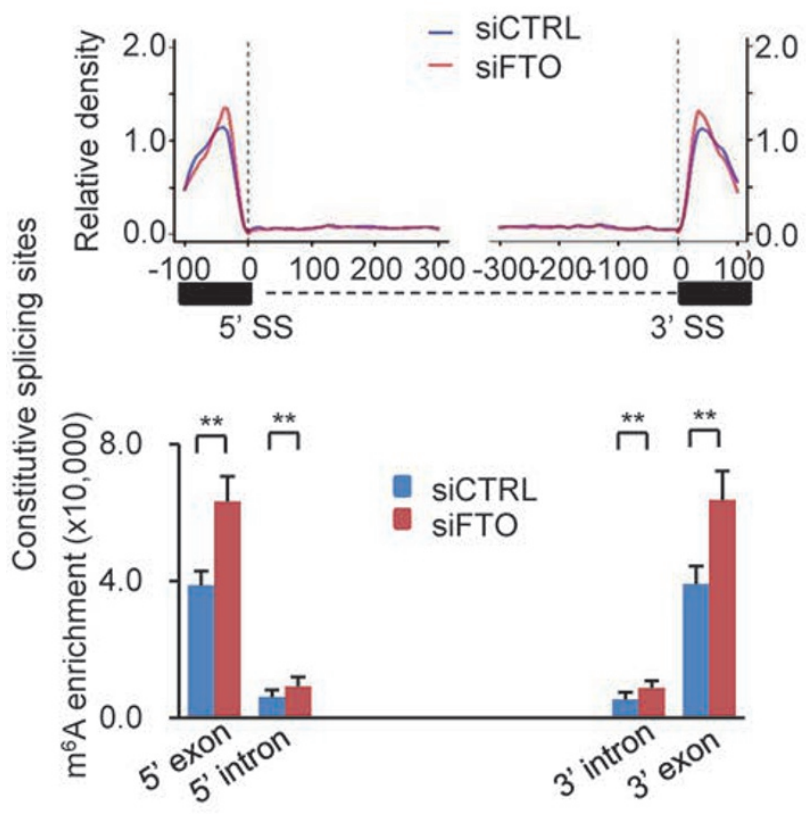

C

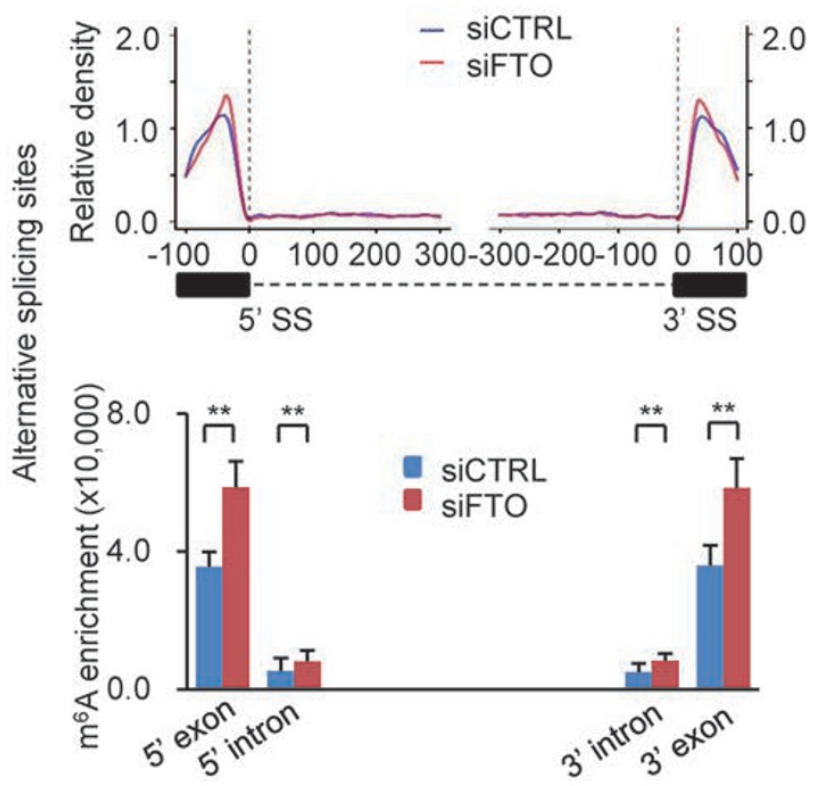

B

FTO target genes (452 genes)
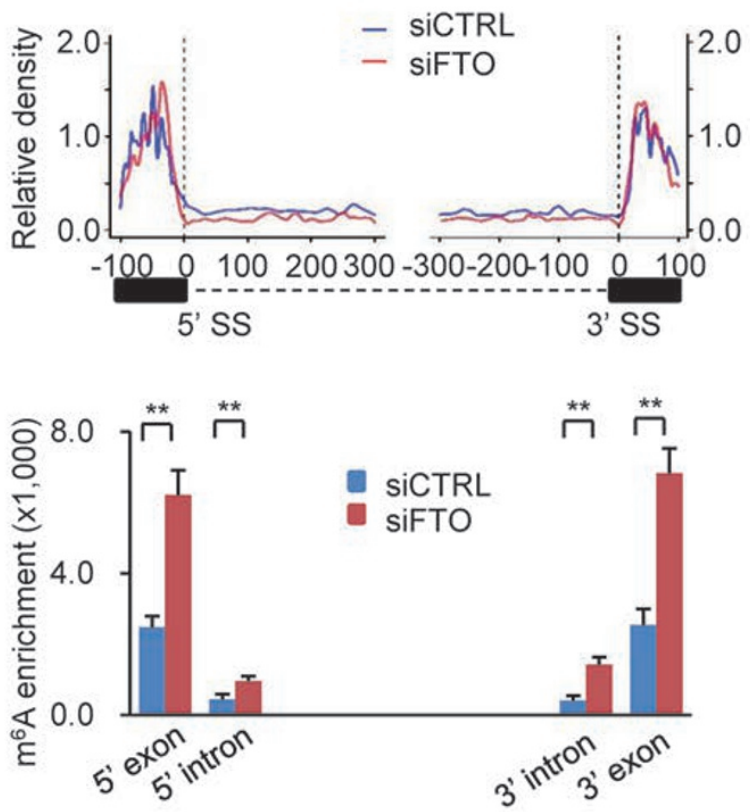

D FTO target genes (452 genes)
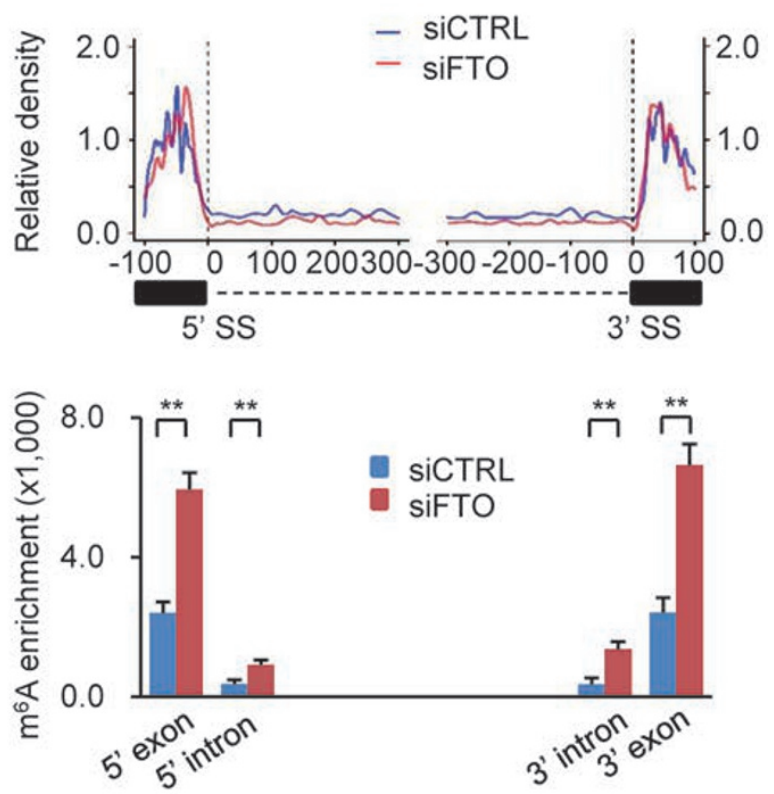

Figure $3 \mathrm{~m}^{6} \mathrm{~A}$ is enriched at exonic splice sites. (A-D) Schematic analysis of the distribution of $\mathrm{m}^{6} \mathrm{~A}$ peaks (upper panel in each figure; $100 \mathrm{nt}$ upstream and $300 \mathrm{nt}$ downstream from 5'SS; $300 \mathrm{nt}$ upstream and $100 \mathrm{nt}$ downstream from 3'SS) and $\mathrm{m}^{6} \mathrm{~A}$ enrichment (lower panel in each figure; $100 \mathrm{nt}$ up/downstream of the $5^{\prime} \mathrm{SS}$ or $3^{\prime} \mathrm{SS}$ ) along $5^{\prime}$ exon-intron and $3^{\prime}$ intron-exon boundaries in control (blue) and FTO-deficient (red) cells. (A, C) $\mathrm{m}^{6} \mathrm{~A}$ density in the vicinity of constitutive SS (A) or alternative SS (C) in the 5326 genes showing changes in isoform expression upon FTO depletion. (B, D) Similar to A and C but here the $\mathrm{m}^{6} \mathrm{~A}$ density was determined in the 452 FTO target genes showing increased $\mathrm{m}^{6} \mathrm{~A}$ levels after FTO knockdown. The $\mathrm{m}^{6} \mathrm{~A}$ levels in each region between control and FTO depletion are shown as mean \pm SD and statistical analysis on their difference were performed by Student's $t$-test. ${ }^{* *} P<0.01$. See also Supplementary information, Figures S4 and S5. 
A
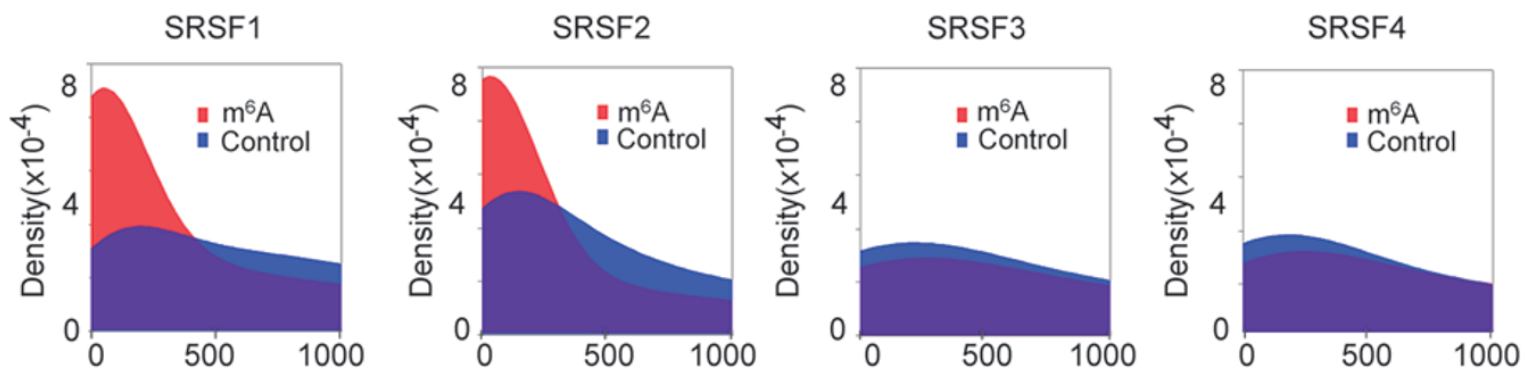

Distance to $\mathrm{m}^{6} \mathrm{~A}$ site $(\mathrm{nt})$

B

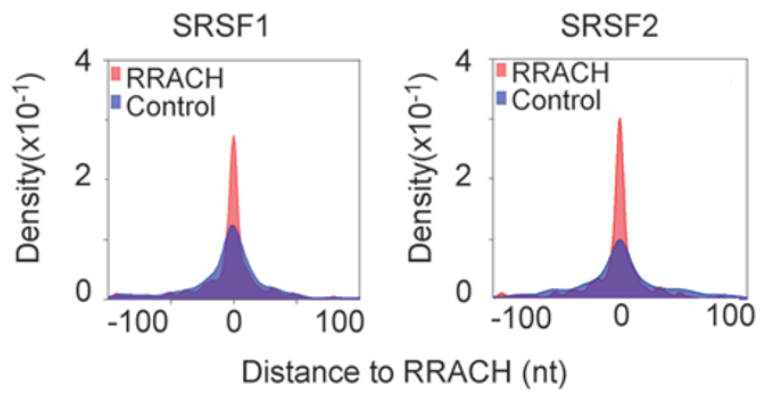

D

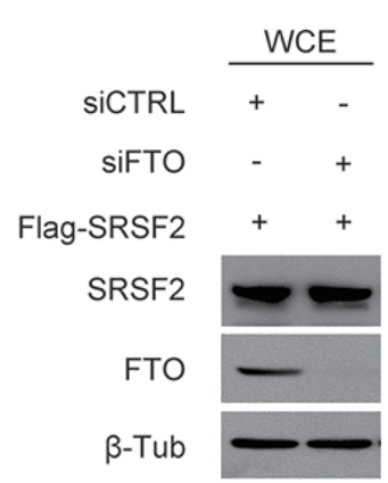

C

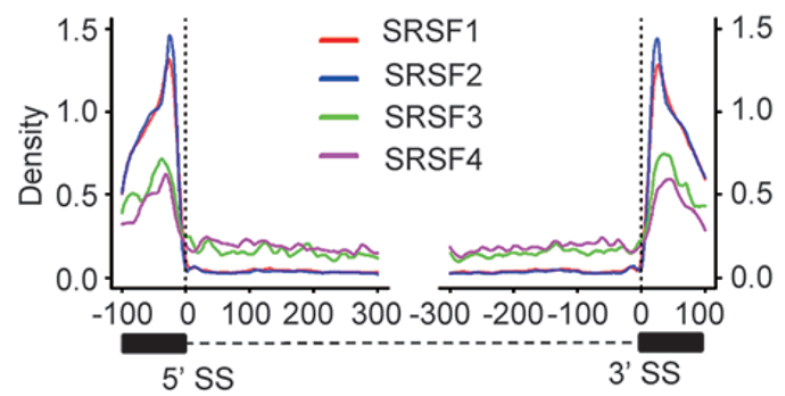

E

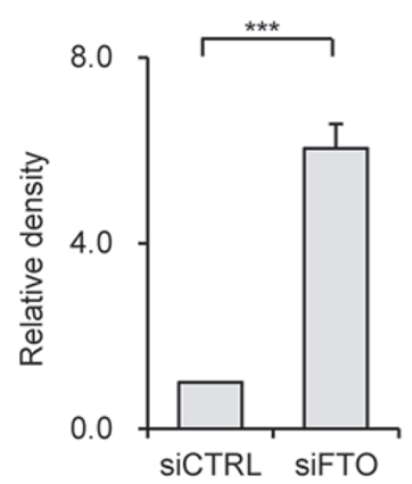

Figure $4 \mathrm{~m}^{6} \mathrm{~A}$ modification influences RNA binding ability of SRSF2. (A) Density plots showing the relative distance (X-axis), calculated by BEDTools' closestBed, between $\mathrm{m}^{6} \mathrm{~A}$ sites and SRSF1-4-binding sites. Randomly selected sites (blue color) within the same co-regulated genes were used as control. (B) The distance between the top-ranking SRSF1 and 2 ESEs predicted by ESEfinder and the nearest RRACH in identified $\mathrm{m}^{6} \mathrm{~A}$ peak (red color) were calculated by BEDTools' closestBed ( $\mathrm{X}$ axis). The distances of $\mathrm{m}^{6} \mathrm{~A}$-modified RRACH sites to the ESEs predicted by ESEfinder were calculated and their relative density distributions were shown as density plot. Randomly selected sites (blue color) within the same co-regulated genes were used as control. (C) Analysis of the distribution of SRSF1-4-binding clusters along 5' exon-intron and 3' intron-exon boundaries (100 nt upstream and $300 \mathrm{nt}$ downstream of 5'SS; $300 \mathrm{nt}$ upstream and $100 \mathrm{nt}$ downstream of 3'SS). (D) PARCLIP of SRSF2 in control and FTO-depleted 3T3-L1 cells transfected with the pCS2-Flag-SRSF2 plasmid. SRSF2 protein-RNA complex was pulled down with Flag M2 Affinity Gel (mouse), and then RNA was labeled and detected following instruction of biotin labeling kit. The expression levels of SRSF2 protein in whole cell lysate (WCE) was detected by western blotting with Flag antibody (rabbit). Knockdown efficiency of FTO was detected with its specific antibody. $\beta$-tubulin was used as loading control. (E) RNA binding ability of SRSF2 was calculated by normalizing binding RNAs to the corresponding pulldowned proteins. Statistical analysis shows the relative binding ability of SRSF2 in FTO-depleted cells $(P<0.0001)$ to that in control cells (siCTRL). See also Supplementary information, Figures S6-S7. 
Figure S7C and S7D). As FTO does not interact with SRSF2 (data not shown), changes in RNA binding ability of SRSF2 cannot be explained by potential changes in direct protein-protein recruitment of SRSF2 by FTO. Taken together, these results suggest that $\mathrm{m}^{6} \mathrm{~A}$ may serve as a new exonic cis-regulatory signal for RNA splicing, which together with ESEs co-regulates SRSF2 protein recruitment.

\section{$M^{6} A$ modification regulates splicing of SRSF2 target genes}

To test our above hypothesis, we chose alternatively spliced genes that contain spatially overlapped SRSF2-binding sites and $\mathrm{m}^{6} \mathrm{~A}$ sites around spliced exons (including $5^{\prime}$ and $3^{\prime}$ flanking exons and internal alternative exons), for alternative splicing analysis by RT-PCR. A representative gene FBXO9 showed increased $\mathrm{m}^{6} \mathrm{~A}$ levels around splicing site upon FTO depletion (Supplementary information, Figure S4). We found that FTO depletion inhibited exon skipping and promoted inclusion of the alternative exons of GKAP1, ZFP809, FBXO9, KIF13A and ZFP212 genes, as shown by the decreased exclusion levels (Figure 5A, 5B and Supplementary information, Figure S6E). Supporting our findings, previously published CLIP-seq data showed that SRSF2 binds to exons around alternative splice sites of these genes [45].

We performed $\mathrm{m}^{6} \mathrm{~A}-\mathrm{IP}$ and PAR-CLIP in control and FTO-depleted 3T3-L1 cells. All of the tested spliced genes showed increased $\mathrm{m}^{6} \mathrm{~A}$ levels upon FTO depletion, supporting the notion that they are direct targets of FTO (Figure 5C). Consistent with the global effect of FTO depletion on the binding ability of SRSF2, binding of SRSF2 to above tested genes increased when FTO was knocked down (Figure 5D). SRSF2 is a splicing regulator reported to influence RNA splicing. When SRSF2 binding exceeds a certain level, inclusion of the alternative exons is ensured [45]. Hence, the increased binding ability of SRSF2 to RNA supported by increased $\mathrm{m}^{6} \mathrm{~A}$ levels may promote exon inclusion in alternative splicing of FTO target genes.

We further confirmed the splicing events in METTL3-depleted 3T3-L1 cells. Exon exclusion levels of FBXO9, KIF13A and ZFP212 increased upon METTL3 knockdown (Supplementary information, Figure S8AS8C). The opposite responses of the splicing events in FTO- and METTL3-depleted cells indicate that they are under the direct influence of $\mathrm{m}^{6} \mathrm{~A}$ dynamics. Next, we asked whether these splicing events were SRSF2-dependent. We examined this hypothesis in SRSF2-depleted 3T3-L1 cells. Exon exclusion levels of FBXO9 and KIF13A were increased in response to SRSF2 deletion, opposite to the effects of FTO depletion (Supplementary information, Figure S8D and S8E).

FTO regulates alternative splicing of RUNX1T1 which modulates preadipocyte diffentiation.

To validate the biological relevance of $\mathrm{m}^{6} \mathrm{~A}$-dependent regulation of SRSF2 binding and mRNA splicing, we chose to thoroughly examine the expression and function of an adipogenesis-related transcription factor, Runt-related transcription factor 1 (RUNX1T1) [52]. RUNX1T1 can be expressed in two isoforms. The PCR product of the constitutive isoform RUNX1T1-L is $496 \mathrm{bp}$, while that of the exon 6-skipped isoform RUNX1T1-S is 245 bp (Figure 6A). The PCR products were verified by sequencing analysis (Supplementary information, Figure S9A). In FTO-depleted 3T3-L1 cells, the exclusion level of exon 6 was decreased (Figure 6A and 6B). To the contrary, the exclusion level was increased in METTL3-depleted 3T3-L1 cells (Supplementary information, Figure S9B and S9C).

We applied a commercially available antibody recognizing both RUNX1T1 isoforms to verify that the mRNA expression pattern corresponded to what could be observed at the protein level. The RUNX1T1-L protein $(68 \mathrm{kDa})$ was equally expressed in both control and FTO-depleted cells, while only very little amount of RUNX1T1-S (27 kDa) could be detected in FTO-depleted cells compared with control cells (Figure 6C and 6D). FTO depletion resulted in increased $\mathrm{m}^{6} \mathrm{~A}$ levels in exons 5-7 (Figure 6E), which was accompanied by significant increase in SRSF2 binding (Figure 6F), suggesting that FTO may control the alternative splicing of RUNX1T1 by regulating $\mathrm{m}^{6} \mathrm{~A}$ levels and thus SRSF2 accumulation around the splice sites. The finding that RUNX1T1 isoform expression is regulated by FTO prompted us to explore the potential role of RUNX1T1-L and -S in adipogenesis.

During adipocyte differentiation, RUNX1T1-S expression displays a progressive decrease as that of FTO (Supplementary information, Figure S9D and S9E). This result is in consistent with the observed splicing pattern of RUNX1T1 upon FTO knockdown. Oil Red O staining and triglyceride assays showed a significant inhibition of differentiation by RUNX1T1-L, whereas RUNX1T1-S appeared to promote differentiation (Figure 6G-6I), suggesting that FTO via its regulation of $\mathrm{m}^{6} \mathrm{~A}$ levels and SRSF2 binding controls RUNX1T1 isoform expression, which in turn contributes to the regulation of adipogenesis. The significantly reduced expression of RUNX1T1-S might partially explain the differentiation defect observed in FTO-deficient cells. 
A
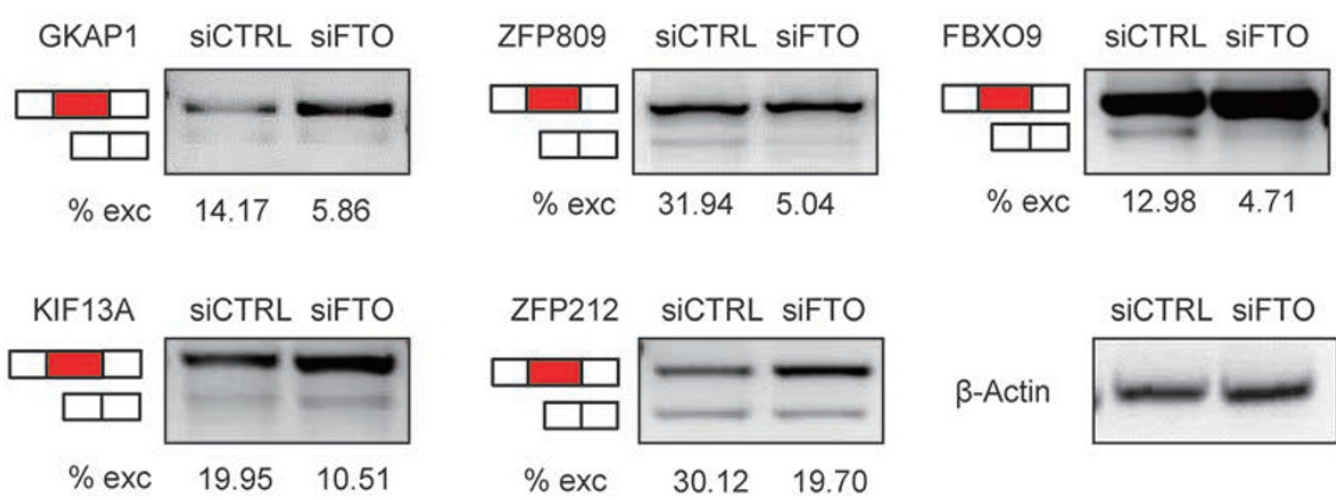

B

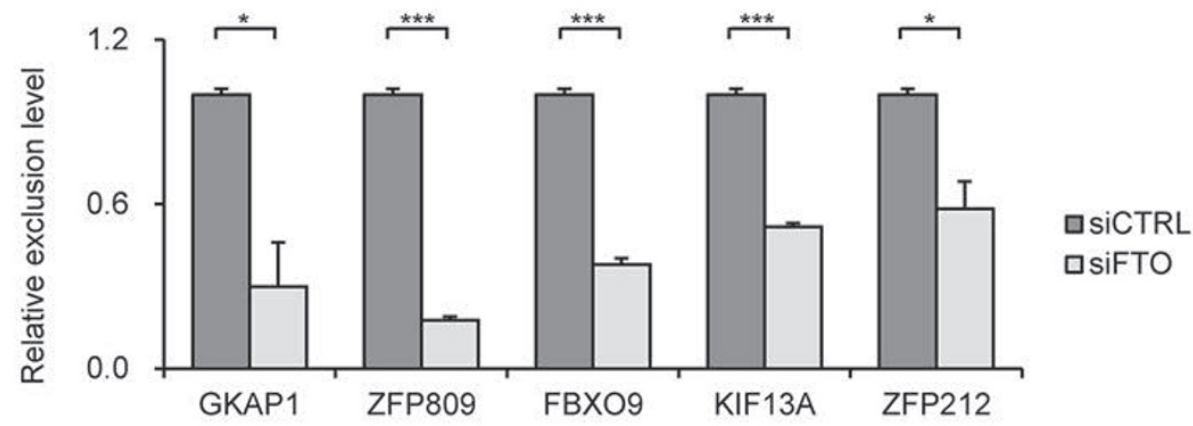

C

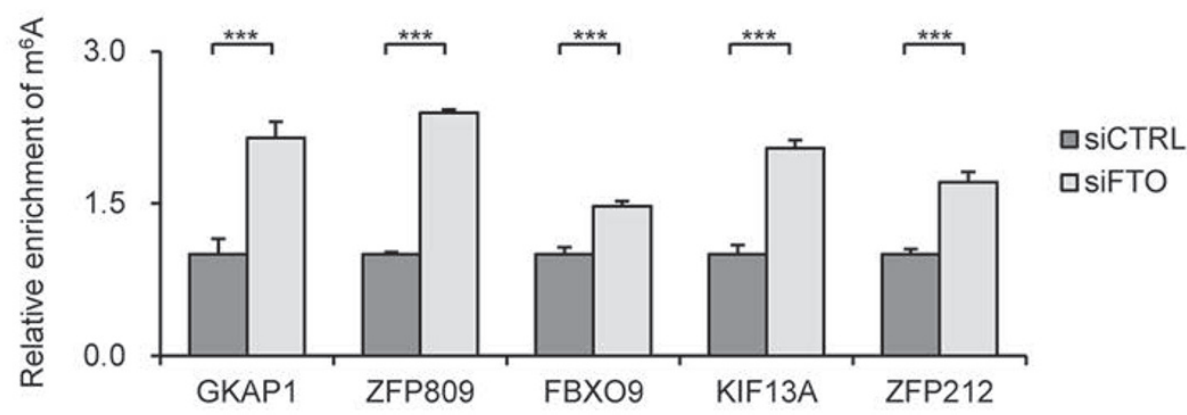

D

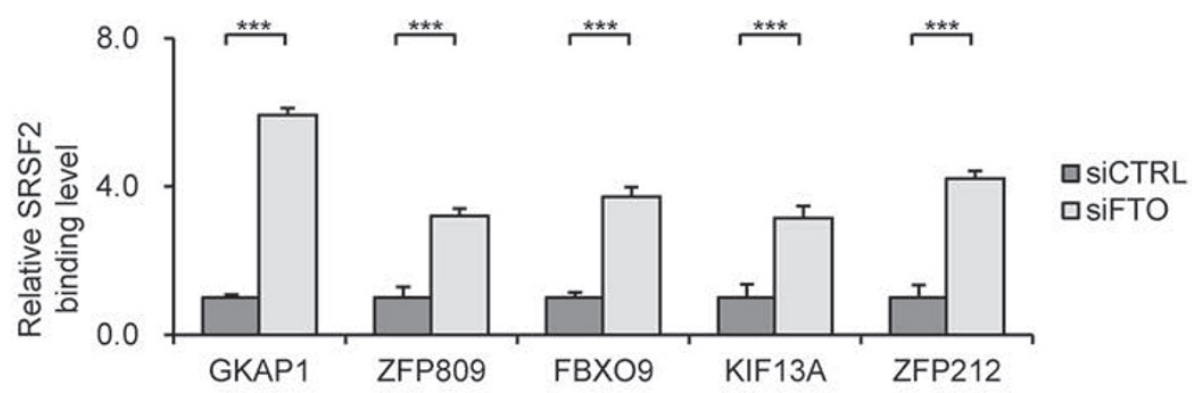

Figure 5 FTO regulates splicing in a subset of SRSF2 target genes. (A) Splicing analysis of SRSF2 target genes by RT-PCR in FTO-depleted cells. Red boxes indicate the spliced internal exons. \% exc (exclusion) indicates the exclusion level of the internal exons. The expression of Actin was detected to confirm the equal loading of PCR products. (B) Statistical analysis of the relative exclusion level of spliced exons in FTO-depleted 3T3-L1 cells to that in control cells. ${ }^{* * *} P<0.0001,{ }^{*} P<0.05$. Results are shown as mean \pm SD. (C) Detection of $m^{6} A$ levels around splicing sites of FTO target genes by $m^{6} A-I P$ and RT-qPCR. ${ }^{* * *} P<$ 0.0001. Results are shown as mean \pm SD. (D) Effects of FTO depletion on SRSF2 binding ability to its target genes validated by PAR-CLIP and RT-qPCR. ${ }^{* * *} P<0.0001$. Results are shown as mean \pm SD. See also Supplementary information, Figure S8. 


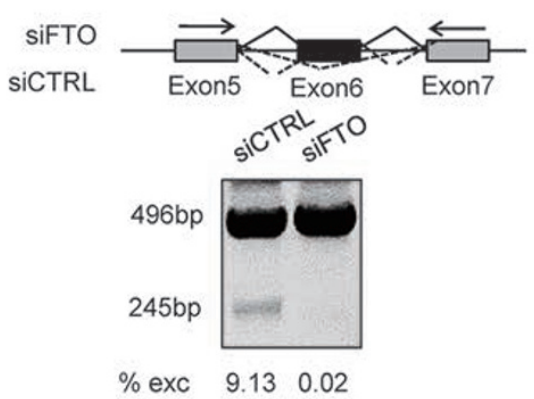

C

D
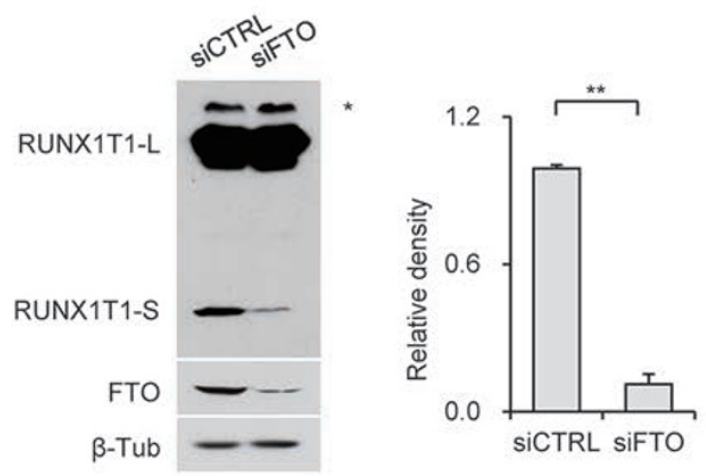

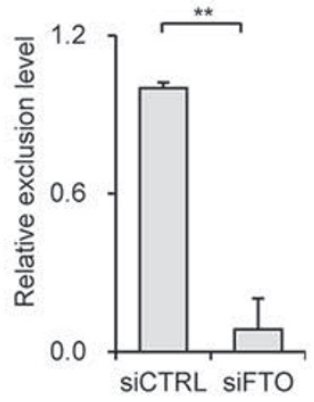

E

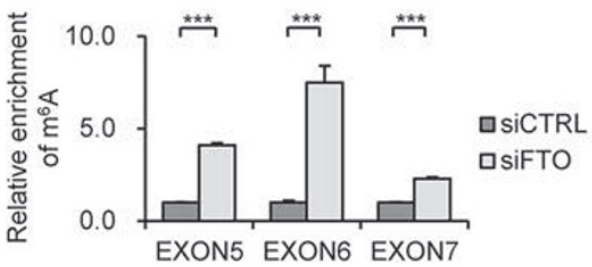

F

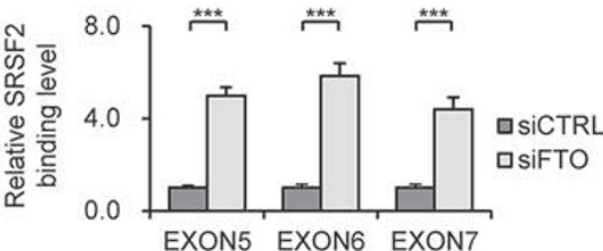

G

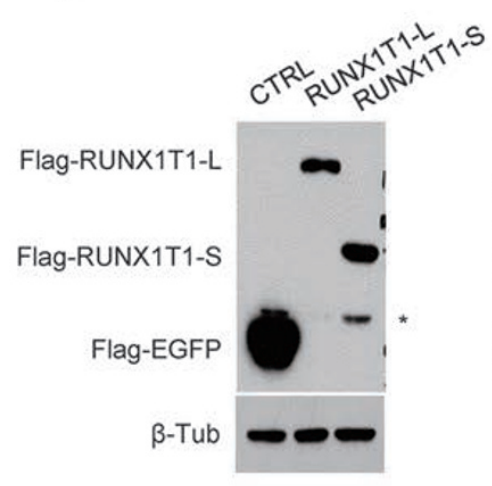

H

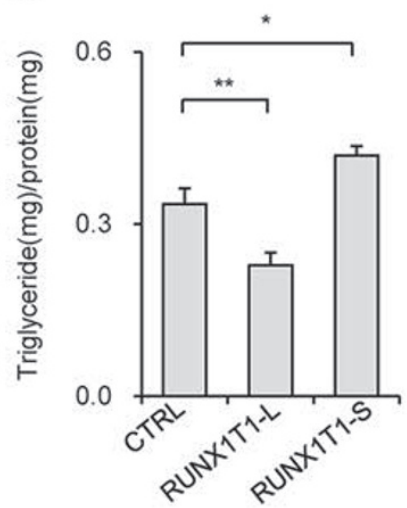

I

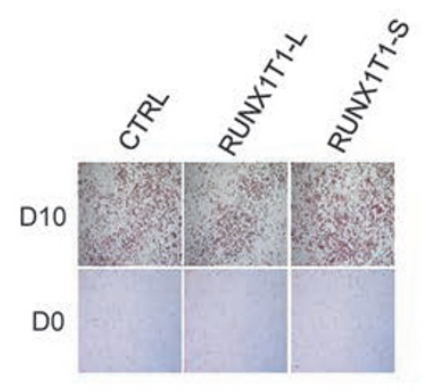

Figure 6 FTO regulates alternative splicing of adipogenensis-related factor RUNX1T1. (A) Alternative splicing of RUNX1T1 was identified by RT-PCR using the indicated PCR primer set (arrows). Depending on isoform, bands of either 496 bp or $245 \mathrm{bp}$ can be detected. (B) Statistical analysis of the relative exclusion level of RUNX1T1 in FTO-depleted 3T3-L1 cells to that in control cells. ${ }^{* *} P<0.01$. Results are shown as mean \pm SD. (C) RUNX1T1 detection in 3T3-L1 pre-adipocyte lysates shows the endogenous protein products of the two RUNX1T1 isoforms in control and FTO-deficient 3T3-L1 pre-adipocytes. An unspecific band was labeled by *. (D) Statistical analysis of RUNX1T1-S protein expression levels in FTO-depleted 3T3L1 cells to that in control cells. ${ }^{* *} P<0.01$. Results are shown as mean $\pm \mathrm{SD}$. (E) Detection of $\mathrm{m}^{6} \mathrm{~A}$ levels around splicing sites of RUNX1T1 gene by $\mathrm{m}^{6} \mathrm{~A}-\mathrm{IP}$ and RT-qPCR. ${ }^{* *} P<0.0001$. Results are shown as mean $\pm \mathrm{SD}$. (F) Effect of FTO depletion on SRSF2 binding ability to exons around splicing sites validated by PAR-CLIP and RT-qPCR. ${ }^{* * *} P<0.0001$. Results are shown as mean \pm SD. (G-I) 3T3-L1 pre-adipocytes were transfected with pEGFP-C1b, pEGFP-C1b-Runx1T1-L or pEGFPC1b-Runx1T1-S. Fory-eight hours later, transfection cells were lysed and immunoblotted with the indicated antibodies (G). An unspecific band or degradation band was labeled by *. Transfected cells were induced to differentiation and subjected to triglyceride analysis $(\mathbf{H})$ and Oil Red O staining (I) on D10. Triglyceride content was quantified and normalized to protein content. ${ }^{*} P<0.05$ is considered significant. ${ }^{*} P<0.01$. Results are shown as mean \pm SD. See also Supplementary information, Figure S9. 


\section{Discussion}

In this study we demonstrate that FTO contributes to the regulation of mRNA alternative splicing by modulating $\mathrm{m}^{6} \mathrm{~A}$ levels and hence SRSF2 binding at splice sites. We show a novel FTO-dependent function of $\mathrm{m}^{6} \mathrm{~A}$ in the regulation of mRNA splicing in adipocytes and in the process of adipogenesis. There are several pieces of evidence suggesting a role of $\mathrm{m}^{6} \mathrm{~A}$ in RNA splicing: $\mathrm{m}^{6} \mathrm{~A}$ are preferentially found in exons and UTR regions in multi-isoform genes and alternatively spliced exons [18], binding of the $\mathrm{m}^{6} \mathrm{~A}$ methyltransferase complex is especially enriched in genes with multiple isoforms and alternatively spliced exon junctions [27], and finally, depletion of either METTL3 or ALKBH5 causes mRNA splicing alterations $[18,34]$. However, it has remained unclear how $\mathrm{m}^{6} \mathrm{~A}$ may contribute to the regulation of mRNA alternative splicing. The pronounced enrichment of $\mathrm{m}^{6} \mathrm{~A}$ in exonic sequences spatially overlapping with ESEs and binding clusters of specific SR proteins, together with the finding that $\mathrm{m}^{6} \mathrm{~A}$ regulates RNA binding of SRSF2, suggests that $\mathrm{m}^{6} \mathrm{~A}$ might influence the choice of exon inclusion/exclusion by regulating SRSF2 access to its targets. The accurate recognition of exon/intron boundaries is essential for correct splicing and generation of mature mRNA. In higher eukaryotes, exon-intron junctions are defined by conserved intronic cis-elements, including the $5^{\prime}$ splice site and $3^{\prime}$ splice site. Exonic cis-elements such as exonic splicing enhancers (ESEs) or silencers (ESSs) are important for correct splice-site identification and prevalent in most exons, including constitutive ones. ESEs serve as binding sites for specific serine/arginine-rich (SR) proteins. ESEs and their binding SR proteins frequently exist in protein-coding exons, compared with flanking intronic regions [53]. Similarly, in CDS regions, $\mathrm{m}^{6} \mathrm{~A}$ is highly enriched at the edges of exons adjacent to splice sites relative to intron regions. Knockdown of FTO specifically affects the RNA binding ability of SRSF2, but not that of SRSF4. This might not be surprising since SRSF2 readily binds to exons in contrast to other SR proteins such as SRSF3 and SRSF4 which have been reported to preferentially bind to intronless genes, ncRNAs and introns or 3'-UTRs of intron-containing genes $[45,46,54]$. Hence $\mathrm{m}^{6} \mathrm{~A}$ dynamics appears to specifically affect the RNA binding ability of SRSF2, thus specifically influencing the splicing outcome of genes under the regulation of SRSF2 (Figures 5 and 6), which can promote both inclusion and skipping of exons [45]. However, in the case of $\mathrm{m}^{6} \mathrm{~A}$-dependent SRSF2 regulation of mRNA splicing, SRSF2 appears to exclusively promote constitutive splicing (inclusion of exons) while inhibiting alternative splicing (exclusion of exons). Thus $\mathrm{m}^{6} \mathrm{~A}$ seems to bring another layer of regu- lation to the mRNA splicing process (Figure 7). A thorough mechanistic understanding of the interplay between $\mathrm{m}^{6} \mathrm{~A}, \mathrm{ESEs}$ and SR proteins will be a challenge for future studies.

FTO has for a long time been implicated in obesity. Our findings show a clear connection between FTO-dependent $\mathrm{m}^{6} \mathrm{~A}$ regulation and the ability of pre-adipocytes to differentiate. It appears, at least in part, to be due to the apparently very different regulatory functions of the two alternative RUNX1T1 isoforms in adipogenesis. Our finding that FTO regulates the splicing of a subset of genes, such as sterol metabolic process-related genes, via controlling $\mathrm{m}^{6} \mathrm{~A}$ levels (Figure $2 \mathrm{G}$ ), may help explain why FTO appears to play a role in obesity [55]. We believe it is reasonable to imagine that changes in the ratio of specific isoforms might have dramatic impact on not only adipogenesis and obesity but also a wide range of biological functions. The finding of a specific role of FTO but not ALKBH5 suggests that these two demethylases function in different physiological processes likely by regulating different subsets of mRNAs.

In summary, we demonstrate a novel FTO-dependent regulatory role of $\mathrm{m}^{6} \mathrm{~A}$ and SRSF2 in mRNA splicing and adipocyte differentiation. The dynamic regulation of $\mathrm{m}^{6} \mathrm{~A}$ by FTO in adipocytes is important in the determination of splicing and gene expression patterns in a subset of genes contributing to the regulation of adipogenesis. The spatial overlap of $\mathrm{m}^{6} \mathrm{~A}$ sites with splice sites and cis-regulatory elements as well as the apparent regulation of SRSF2 binding by $\mathrm{m}^{6} \mathrm{~A}$ call for a further elucidation of the role of $\mathrm{m}^{6} \mathrm{~A}$ in RNA splicing. The direct connection between isoform expression of the adipogenic regulatory factor RUNX1T1 and its $\mathrm{m}^{6} \mathrm{~A}$ status in the context of adipocyte differentiation sheds new light on our understanding of the biological significance of RNA $\mathrm{m}^{6} \mathrm{~A}$ modification.

\section{Materials and Methods}

\section{Cell line, plasmids and antibodies}

3T3-L1 pre-adipocytes were obtained from ATCC (Manassas, VA). The human FTO and mouse RUNX1T1 genes were cloned into pEGFP-C1B [56]. Human SR plasmids were bought from OriGene Technologies (Rockville, MD). The expression constructs were generated using PCR and subcloned into pCS2+ vectors with an N-terminal FLAG tag [57]. Polyclonal rabbit anti-FTO antibody was affinity-purified from rabbits immunized with $6 \times \mathrm{His}-$ tagged full-length human FTO protein as previously reported [9]. Polyclonal rabbit anti-ALKBH5 antibody was generated against synthesized peptide (RKYQEDSDERSD, 58-70 amino acids of human ALKBH5) by CWBio (Beijing) as previously reported [34]. The primary antibodies were purchased from commercial sources: rabbit anti-METTL3 (MT-A70; 15073-1-AP, Proteintech Group); rabbit anti-RUNX1T1 (15494-1-AP, Proteintech); mouse anti- $\beta$-tubulin (t-5293, Sigma); rabbit anti-m ${ }^{6}$ A (202003; Synaptic 


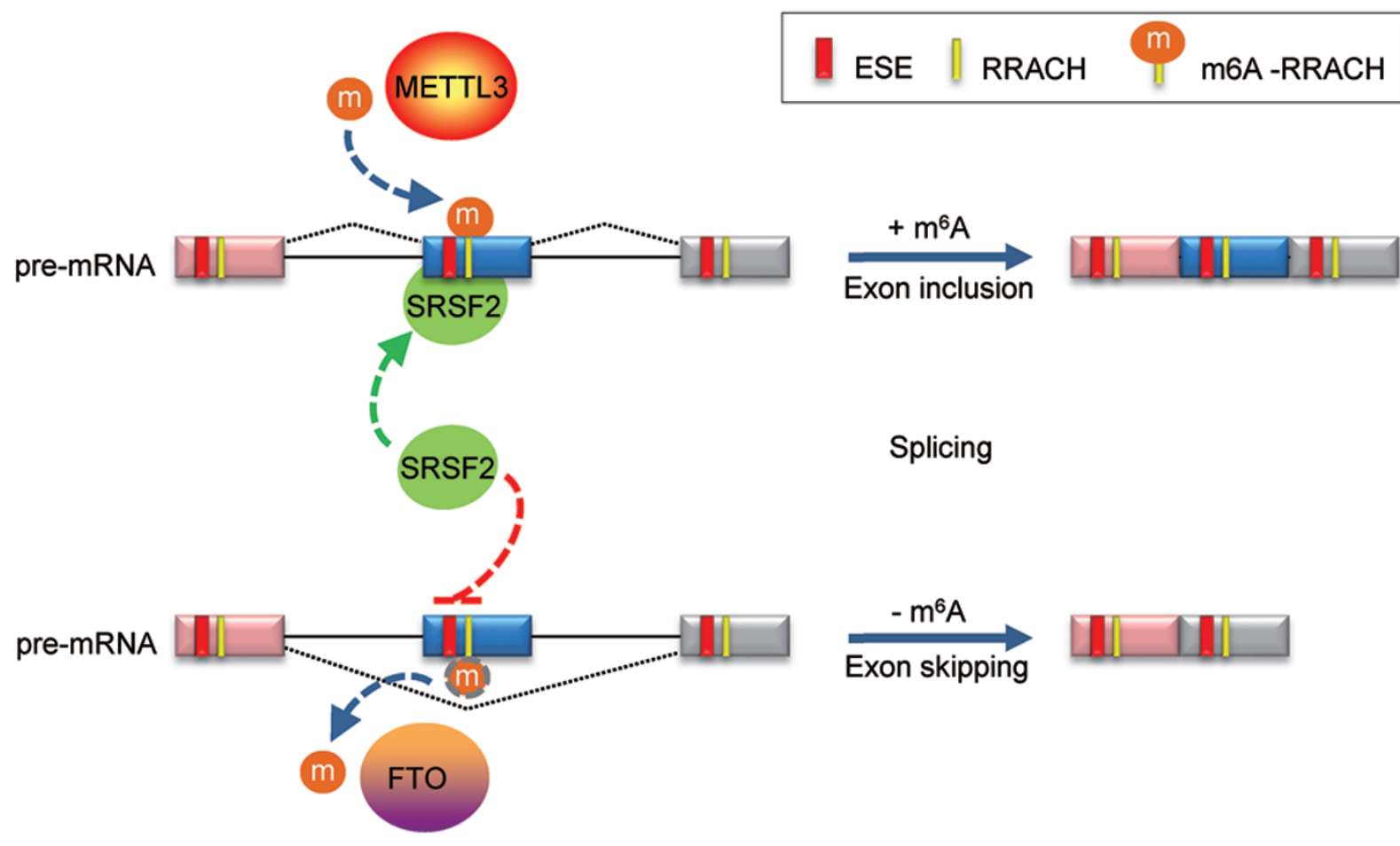

Figure 7 Cooperative role of $\mathrm{m}^{6} \mathrm{~A}$ in regulating SRSF2 function at splice sites. The splicing factor SRSF2 can recognize exon splicing enhancer (ESE), inducing exon inclusion. METTL3-mediated $\mathrm{m}^{6} \mathrm{~A}$ modification enhances the recruitment of SRSF2 to its target ESE, promoting exon inclusion. On the other hand demethylation of $\mathrm{m}^{6} \mathrm{~A}-\mathrm{RRACHs}$ near ESEs by FTO prevents recognition of the ESEs by SRSF2, thereby inhibiting inclusion and instead promoting exon skipping of the specific exon. $\mathrm{M}^{6} \mathrm{~A}$ co-regulated by methyltransferases and demthylases serves as a new RNA splicing exonic cis-regulatory element that in combination with ESEs regulates SRSF2 protein recruitment.

Systems); mouse anti-HA (cw0092B, CWbiotech); rabbit anti-Flag (F7425, Sigma); mouse anti-Flag (F1804, Sigma). The secondary antibodies used for immunoblotting and dot-blotting: anti-Rabbit IgG-HRP (p0448, dakocytomation); anti-Mouse IgG-HRP (p0161, dakocytomation).

\section{Cell culture and adipocyte differentiation}

3T3-L1 pre-adipocytes were grown in DMEM Dulbecco's Modified Eagle Medium (DMEM) containing 10\% newborn calf serum (SH30401.01, Hyclone) and 1\% antibiotics until confluence and induced to differentiation as previously described. In brief, two days' post-confluence (day 0, D0), cells were exposed to differentiation medium containing $0.5 \mathrm{mmol} / \mathrm{L}$ isobutylmethylxanthine (I5879, Sigma), $1 \mu \mathrm{mol} / \mathrm{L}$ dexamethasone (D2915, Sigma), $10 \mu \mathrm{g} / \mathrm{mL}$ insulin (I6634, Sigma), $2 \mu \mathrm{mol} / \mathrm{L}$ rosiglitazone (R2408, Sigma) and $10 \%$ fetal bovine serum (FBS; A15-151, PAA) for three days. At the end of day 3 , culture medium was replaced with DMEM supplemented only with $10 \mu \mathrm{g} / \mathrm{mL}$ insulin and $10 \% \mathrm{FBS}$, and replenished every other day. After the differentiation process, at least $90 \%$ of the cells had accumulated lipid droplets at day 10 , and were used as mature adipocytes.

\section{Gene expression and splicing analyses}

Total RNA was isolated from cells with TRI Reagent (Sigma). cDNA library was constructed using TruSeq. RNA Sample Prep
Kit and then sequenced with HiSeq 2000 system (Illumina Inc.). RNA-seq reads were mapped to the mouse reference genome (mm10) by Tophat (V2.0.4) [58, 59]. Number of reads mapped to each Ensembl gene were counted using the HTSeq python package and the DEGs in different samples were determined by R-package DEGseq [60]. The expressions of transcripts were quantified as Reads Per Kilobase of exon model per Million mapped reads (RPKM).

For splicing analysis, spliced events were identified and compared with Cufflink and Scripture softwares [61, 62]. Cuffdiff, one part of the Cufflinks package were applied to calculate the reads count and FPKM for each isoform of each gene. The differentially expressed isoforms were identified using R-package DEGseq. Scripture was used to detect splicing events within each gene [62]. Differentially expressed exons analysis was done by the R package DEXSeq [63]. To differentially expressed introns, the number of reads falling in each intron were counted using RSeQC [64], and differential expression analysis was done using the DEGseq [60]. The exon-exon junctions approach applied in previous study was also used to analyze alternative splicing event [65].

\section{$M^{6} A$-seq analyses}

mRNA was extracted using biotinylated poly(dT) oligo, followed by further removing of contaminated rRNA using RiboMinus Transcriptome Isolation Kit (K1550-02, Invitrogen). The 
mRNA quality was analyzed by NanoDrop. mRNA was fragmented and subjected to immunoprecipitation with $\mathrm{m}^{6} \mathrm{~A}$ specific antibody as previously described approaches $[17,18] . \mathrm{M}^{6} \mathrm{~A}$-enriched RNA was used to generate the cDNA library in parallel with the input RNA, and then sequenced using the HiSeq 2000 system (Illumina Inc.). For $\mathrm{m}^{6} \mathrm{~A}$ analysis, $\mathrm{m}^{6} \mathrm{~A}$ regions $\left(\mathrm{m}^{6} \mathrm{~A}\right.$ peaks) were identified by comparing the read abundance between $\mathrm{m}^{6} \mathrm{~A}$-seq and RNA-seq samples of the same loci according to a method previously described [17]. Briefly, the entire mm10 genome was divided into $25 \mathrm{nt}$ bins and the numbers of both $\mathrm{m}^{6} \mathrm{~A}$-seq reads and RNA-seq reads (used as control) mapped to each bin were counted and compared. Bins with statistically enriched $\mathrm{m}^{6} \mathrm{~A}$-seq reads compared with the RNA-seq reads (adjusted $P<1 \mathrm{e}-5$, Fisher's exact test together with Benjamini-Hocberg procedure) were identified and concatenated adjacently. Using the same selection criteria, regions with significantly enriched RNA-seq reads were selected as control peaks. Sequence motifs enriched in $\mathrm{m}^{6} \mathrm{~A}$ peaks were identified by HOMER with $\mathrm{m}^{6} \mathrm{~A}$ peaks as the target sequences and control peaks as the background [66]. The spatial correlations between $\mathrm{m}^{6} \mathrm{~A}$ sites (RRACH) and ESEs or binding clusters of SR proteins were analyzed by BEDTools software [67].

\section{PAR-CLIP}

3T3-L1 cells were co-transfected with pCS2-SR plamids and control siRNAs and FTO siRNAs, respectively. Thirty-two hours later, cells were labeled with $200 \mu \mathrm{M}$ 4-SU (Sigma T4509) for 16 $\mathrm{h}$, then to induce crosslinking treated with $0.4 \mathrm{~J} / \mathrm{cm}^{2}$ of $365 \mathrm{~nm} \mathrm{UV}$ light in a BLX-E365 crosslinker BLX-E365 (Vilber). Irradiated cells were then lysed with NP-40 lysis buffer (50 mM pH 7.5 Tris$\mathrm{Cl}, 150 \mathrm{mM} \mathrm{NaCl}, 2 \mathrm{mM}$ EDTA, $0.5 \%$ (v/v) NP40) on ice for 30 min. Cell debris was removed by centrifugation and the crude lysates were incubated with Flag M2 Affinity Gel (A2220, Sigma) for $2 \mathrm{~h}$ at $4{ }^{\circ} \mathrm{C}$. The immunoprecipitates were treated with CIP (NEB M0290) and subjected to the biotin labelling, following the instructions of the RNA 3' end biotinylation kit (20160, Thermo). After separation on 4\%-12\% NuPAGE gels (NP0321B0X, Invitrogen), the protein-RNA complexes were transferred to PVDF membranes. Biotin-labelled RNA were detected and visualized according to manufactors description (chemiluminescent nuclei acid kit, Thermo 89880). SR proteins were identified by rabbit Flag antibody (F7425, Sigma).

\section{Accession numbers}

$\mathrm{M}^{6} \mathrm{~A}$-seq and RNA-seq data have been uploaded to GEO database and can be accessed via accession number GSE53244 and GSE53249.

\section{Acknowledgments}

This work was supported by the National Basic Research Program of China (973 Program; 2011CB510103 and 2014CB964902), the National Natural Science Foundation of China (91319308, 31430022, and 31400672), and the Chinese Academy of Sciences (CAS) Strategic Priority Research Program (XDB14030300).

\section{References}

1 Gerken T, Girard CA, Tung YC, et al. The obesity-associated
FTO gene encodes a 2-oxoglutarate-dependent nucleic acid demethylase. Science 2007; 318:1469-1472.

2 Loos RJ, Bouchard C. FTO: the first gene contributing to common forms of human obesity. Obes Rev 2008; 9:246-250.

3 Zabena C, Gonzalez-Sanchez JL, Martinez-Larrad MT, et al. The FTO obesity gene. Genotyping and gene expression analysis in morbidly obese patients. Obes Surg 2009; 19:87-95.

4 Loos RJ, Yeo GS. The bigger picture of FTO - the first GWAS-identified obesity gene. Nat Rev Endocrinol 2014; 10:51-61.

5 Fischer J, Koch L, Emmerling C, et al. Inactivation of the Fto gene protects from obesity. Nature 2009; 458:894-898.

6 Gao X, Shin YH, Li M, Wang F, Tong Q, Zhang P. The fat mass and obesity associated gene FTO functions in the brain to regulate postnatal growth in mice. PLoS One 2010; 5:e14005.

7 Church C, Moir L, McMurray F, et al. Overexpression of Fto leads to increased food intake and results in obesity. Nat Genet 2010; 42:1086-1092.

8 McMurray F, Church CD, Larder R, et al. Adult onset global loss of the fto gene alters body composition and metabolism in the mouse. PLoS Genet 2013; 9:e1003166.

9 Jia GF, Fu Y, Zhao X, et al. N6-Methyladenosine in nuclear RNA is a major substrate of the obesity-associated FTO. Nat Chem Biol 2011; 7:885-887.

10 Jia G, Yang CG, Yang S, et al. Oxidative demethylation of 3-methylthymine and 3-methyluracil in single-stranded DNA and RNA by mouse and human FTO. FEBS Lett 2008; 582:3313-3319.

11 Pan T. N6-methyl-adenosine modification in messenger and long non-coding RNA. Trends Biochem Sci 2013; 38:204209.

12 Niu Y, Zhao X, Wu YS, Li MM, Wang XJ, Yang YG. N6-methyl-adenosine $\left(\mathrm{m}^{6} \mathrm{~A}\right)$ in RNA: an old modification with a novel epigenetic function. Genomics Proteomics Bioinformatics 2013; 11:8-17.

$13 \mathrm{Fu}$ Y, He C. Nucleic acid modifications with epigenetic significance. Curr Opin Chem Biol 2012; 16:516-524.

14 Meyer KD, Jaffrey SR. The dynamic epitranscriptome: N6-methyladenosine and gene expression control. Nat Rev Mol Cell Biol 2014; 15:313-326.

15 Fu Y, Dominissini D, Rechavi G, He C. Gene expression regulation mediated through reversible $\left(\mathrm{m}^{6} \mathrm{~A}\right)$ RNA methylation. Nat Rev Genet 2014; 15:293-306.

16 Bodi Z, Button JD, Grierson D, Fray RG. Yeast targets for mRNA methylation. Nucl Acids Res 2010; 38:5327-5335.

17 Meyer KD, Saletore Y, Zumbo P, Elemento O, Mason CE, Jaffrey SR. Comprehensive analysis of mRNA methylation reveals enrichment in $3^{\prime}$ UTRs and near stop codons. Cell 2012; 149:1635-1646.

18 Dominissini D, Moshitch-Moshkovitz S, Schwartz S, et al. Topology of the human and mouse $\mathrm{m}^{6} \mathrm{~A}$ RNA methylomes revealed by $\mathrm{m}^{6} \mathrm{~A}$-seq. Nature 2012; 485:201-206.

19 Schwartz S, Agarwala SD, Mumbach MR, et al. High-resolution mapping reveals a conserved, widespread, dynamic mRNA methylation program in yeast meiosis. Cell 2013; 155:1409-1421.

20 Wang Y, Li Y, Toth JI, Petroski MD, Zhang ZL, Zhao JC. N-6-methyladenosine modification destabilizes developmen- 
tal regulators in embryonic stem cells. Nat Cell Biol 2014; 16:191-198.

21 Fustin JM, Doi M, Yamaguchi Y, et al. RNA-Methylation-dependent RNA processing controls the speed of the circadian clock. Cell 2013; 155:793-806.

22 Hess ME, Hess S, Meyer KD, et al. The fat mass and obesity associated gene (Fto) regulates activity of the dopaminergic midbrain circuitry. Nat Neurosci 2013; 16:1042-1048.

23 Frayling TM, Timpson NJ, Weedon MN, et al. A common variant in the FTO gene is associated with body mass index and predisposes to childhood and adult obesity. Science 2007; 316:889-894.

24 Zhao X, Yang Y, Sun BF, Zhao YL, Yang YG. FTO and obesity: mechanisms of association. Curr Diab Rep 2014; 14:486.

25 Wang X, Lu Z, Gomez A, et al. N6-methyladenosine-dependent regulation of messenger RNA stability. Nature 2014; 505:117-120.

26 Liu J, Yue Y, Han D, et al. A METTL3-METTL14 complex mediates mammalian nuclear RNA N6-adenosine methylation. Nat Chem Biol 2014; 10:93-95.

27 Ping XL, Sun BF, Wang L, et al. Mammalian WTAP is a regulatory subunit of the RNA N6-methyladenosine methyltransferase. Cell Res 2014; 24:177-189.

28 Schwartz S, Mumbach MR, Jovanovic M, et al. Perturbation of $\mathrm{m}^{6} \mathrm{~A}$ writers reveals two distinct classes of mRNA methylation at internal and 5 sites. Cell Rep 2014; 8:1-13.

29 Long JC, Caceres JF. The SR protein family of splicing factors: master regulators of gene expression. Biochem J 2009; 417:15-27.

30 Martinez-Contreras R, Cloutier P, Shkreta L, Fisette JF, Revil T, Chabot B. hnRNP proteins and splicing control. Advan Experiment Med Biol 2007; 623:123-147.

31 Matlin AJ, Clark F, Smith CW. Understanding alternative splicing: towards a cellular code. Nat Rev Mol Cell Biol 2005; 6:386-398.

32 Braunschweig U, Gueroussov S, Plocik AM, Graveley BR, Blencowe BJ. Dynamic integration of splicing within gene regulatory pathways. Cell 2013; 152:1252-1269.

33 Stoltzfus CM, Dane RW. Accumulation of spliced avian retrovirus mRNA is inhibited in S-adenosylmethionine-depleted chicken embryo fibroblasts. J Virol 1982; 42:918-931.

34 Zheng G, Dahl JA, Niu Y, et al. ALKBH5 is a mammalian RNA demethylase that impacts RNA metabolism and mouse fertility. Mol Cell 2013; 49:18-29.

35 Tews D, Fischer-Posovszky P, Fromme T, et al. FTO deficiency induces UCP-1 expression and mitochondrial uncoupling in adipocytes. Endocrinology 2013; 154:3141-3151

36 Mitterberger MC, Lechner S, Mattesich M, et al. DLK1 (PREF1) is a negative regulator of adipogenesis in CD105(+)/ CD90(+)/CD34(+)/CD31(-)/FABP4(-) adipose-derived stromal cells from subcutaneous abdominal fat pats of adult women. Stem Cell Res 2012; 9:35-48.

37 Wang Y, Sul HS. Pref-1 regulates mesenchymal cell commitment and differentiation through Sox9. Cell Metab 2009; 9:287-302.

38 Min HY, Spiegelman BM. Adipsin, the adipocyte serine protease: gene structure and control of expression by tumor necrosis factor. Nucl Acids Res 1986; 14:8879-8892.

39 Berulava T, Ziehe M, Klein-Hitpass L, et al. FTO levels affect
RNA modification and the transcriptome. Eur J Hum Genet 2013; 21:317-323.

40 Caceres JF, Kornblihtt AR. Alternative splicing: multiple control mechanisms and involvement in human disease. Trends Genet 2002; 18:186-193.

41 Sorek R, Ast G. Intronic sequences flanking alternatively spliced exons are conserved between human and mouse. Genome Res 2003: 13:1631-1637

42 Barash Y, Calarco JA, Gao W, et al. Deciphering the splicing code. Nature 2010; 465:53-59.

43 Wang Y, Gogol-Doring $\mathrm{A}, \mathrm{Hu} \mathrm{H}$, et al. Integrative analysis revealed the molecular mechanism underlying RBM10-mediated splicing regulation. Embo Mol Med 2013; 5:1431-1442.

44 Sanford JR, Coutinho P, Hackett JA, Wang X, Ranahan W, Caceres JF. Identification of nuclear and cytoplasmic mRNA targets for the shuttling protein SF2/ASF. PLoS One 2008; 3:e3369.

45 Pandit S, Zhou Y, Shiue L, et al. Genome-wide analysis reveals SR protein cooperation and competition in regulated splicing. Mol Cell 2013; 50:223-235.

46 Anko ML, Muller-McNicoll M, Brandl H, et al. The RNA-binding landscapes of two SR proteins reveal unique functions and binding to diverse RNA classes. Genome Biol 2012; 13:R17.

47 Konig J, Zarnack K, Rot G, et al. iCLIP reveals the function of hnRNP particles in splicing at individual nucleotide resolution. Nat Struct Mol Biol 2010; 17:909-915.

48 Huelga SC, Vu AQ, Arnold JD, et al. Integrative genome-wide analysis reveals cooperative regulation of alternative splicing by hnRNP proteins. Cell Rep 2012; 1:167-178.

49 Pollard KS, Hubisz MJ, Rosenbloom KR, Siepel A. Detection of nonneutral substitution rates on mammalian phylogenies. Genome Res 2010; 20:110-121.

50 Smith PJ, Zhang C, Wang J, Chew SL, Zhang MQ, Krainer AR. An increased specificity score matrix for the prediction of SF2/ASF-specific exonic splicing enhancers. Hum Mol Genet 2006; 15:2490-2508.

51 Cartegni L, Wang J, Zhu Z, Zhang MQ, Krainer AR. ESEfinder: A web resource to identify exonic splicing enhancers. $\mathrm{Nucl}$ Acids Res 2003; 31:3568-3571.

52 Rochford JJ, Semple RK, Laudes M, et al. ETO/MTG8 is an inhibitor of C/EBPbeta activity and a regulator of early adipogenesis. Mol Cell Biol 2004; 24:9863-9872.

53 Wang J, Smith PJ, Krainer AR, Zhang MQ. Distribution of SR protein exonic splicing enhancer motifs in human protein-coding genes. Nucl Acids Res 2005; 33:5053-5062.

54 Anko ML, Morales L, Henry I, Beyer A, Neugebauer KM. Global analysis reveals SRp20- and SRp75-specific mRNPs in cycling and neural cells. Nat Struct Mol Biol 2010; 17:962970.

55 Dai HJ, Wu JC, Tsai RT, Pan WH, Hsu WL. T-HOD: a literature-based candidate gene database for hypertension, obesity and diabetes. Database (Oxford) 2013; 2013:bas061.

56 Yang YG, Lindahl T, Barnes DE. Trex1 exonuclease degrades ssDNA to prevent chronic checkpoint activation and autoimmune disease. Cell 2007; 131:873-886.

57 Fu Y, Huang B, Shi Z, et al. SRSF1 and SRSF9 RNA binding proteins promote Wnt signalling-mediated tumorigenesis by enhancing beta-catenin biosynthesis. Embo Mol Med 2013; 
5:737-750.

58 Trapnell C, Pachter L, Salzberg SL. TopHat: discovering splice junctions with RNA-Seq. Bioinformatics 2009; 25:1105-1111.

59 Kim D, Pertea G, Trapnell C, Pimentel H, Kelley R, Salzberg SL. TopHat2: accurate alignment of transcriptomes in the presence of insertions, deletions and gene fusions. Genome Biol 2013; 14:R36.

60 Wang L, Feng Z, Wang X, Zhang X. DEGseq: an R package for identifying differentially expressed genes from RNA-seq data. Bioinformatics 2010; 26:136-138.

61 Trapnell C, Williams BA, Pertea G, et al. Transcript assembly and quantification by RNA-Seq reveals unannotated transcripts and isoform switching during cell differentiation. Nat Biotechnol 2010; 28:511-515.

62 Guttman M, Garber M, Levin JZ, et al. Ab initio reconstruction of cell type-specific transcriptomes in mouse reveals the conserved multi-exonic structure of lincRNAs. Nat Biotechnol 2010; 28:503-510.

63 Anders S, Reyes A, Huber W. Detecting differential usage of exons from RNA-seq data. Genome Res 2012; 22:2008-2017.
64 Wang L, Wang S, Li W. RSeQC: quality control of RNA-seq experiments. Bioinformatics 2012; 28:2184-2185.

65 Han H, Irimia M, Ross PJ, et al. MBNL proteins repress ES-cell-specific alternative splicing and reprogramming. $\mathrm{Na}$ ture 2013; 498:241-245.

66 Heinz S, Benner C, Spann N, et al. Simple combinations of lineage-determining transcription factors prime cis-regulatory elements required for macrophage and B cell identities. Mol Cell 2010; 38: 576-589.

67 Quinlan AR, Hall IM. BEDTools: a flexible suite of utilities for comparing genomic features. Bioinformatics 2010; 26:841-842.

(Supplementary information is linked to the online version of the paper on the Cell Research website.)

(c) (i) (3) () This work is licensed under the Creative Commons Attribution-NonCommercial-No Derivative Works 3.0 Unported License. To view a copy of this license, visit http:// creativecommons.org/licenses/by-nc-nd/3.0 